\title{
Thyroid computed tomography imaging: pictorial review of variable pathologies
}

\author{
Mnahi Bin Saeedan ${ }^{1}$ - Ibtisam Musallam Aljohani' ${ }^{2}$ Ayman Omar Khushaim ${ }^{1}$. \\ Salwa Qasim Bukhari ${ }^{3} \cdot$ Salahudin Tayeb Elnaas $^{1}$
}

Received: 7 December 2015 /Revised: 3 May 2016 / Accepted: 24 May 2016 / Published online: 7 June 2016

(C) The Author(s) 2016. This article is published with open access at Springerlink.com

\begin{abstract}
Focal and diffuse thyroid abnormalities are commonly encountered during the interpretation of computed tomography (CT) exams performed for various clinical purposes. These findings can often lead to a diagnostic dilemma, as the CT reflects the nonspecific appearances. Ultrasound (US) examination has a superior spatial resolution and is considered the modality of choice for thyroid evaluation. Nevertheless, CT detects incidental thyroid nodules (ITNs) and plays an important role in the evaluation of thyroid cancer.

In this pictorial review, we cover a wide spectrum of common and uncommon, incidental and non-incidental thyroid findings from CT scans. We also discuss the most common incidental thyroid findings, best practices for their evaluation, and recommendations for their management. In addition, we explore the role of imaging in the assessment of thyroid carcinoma (before and after treatment) and preoperative thyroid goiter, as well as localization of ectopic and congenital thyroid tissue.
\end{abstract}

Mnahi Bin Saeedan

Mbinsaeedan@gmail.com

Department of Radiology, MBC-28, King Faisal Specialist Hospital and Research Center, P.O Box 3354, Riyadh 11211, Saudi Arabia

2 Medical Imaging Department, Ministry of National Guard, Health Affairs, King Abdulaziz Medical City, Riyadh, Saudi Arabia

3 Department of Radiology, University of Tabuk, Tabuk, Saudi Arabia
Teaching Points

- Thyroid disorders tend to have non-specific CTappearances.

- ITNs are common on neck CT.

- ITN management depends on nodule size, age, health status, lymphadenopathy, and invasion.

- $C T$ is used in assessment of cancer extension, mass effect, invasion, and recurrence.

- CT plays a role in preoperative planning in patients with symptomatic goiter.

Keywords Incidental thyroid nodule - Thyroid cancer . Goiter - Ectopic thyroid - Computed tomography

$\begin{array}{ll}\text { Abbreviations } \\ \text { ITN } & \text { Incidental thyroid nodule } \\ \text { CT } & \text { Computed tomography } \\ \text { US } & \text { Ultrasound } \\ \text { FNA } & \text { Fine needle aspiration } \\ \text { IV } & \text { Intravenous } \\ \text { MRI } & \text { Magnetic resonance imaging } \\ \text { PTC } & \text { Papillary thyroid carcinomas } \\ \text { ACR } & \text { American College of Radiology } \\ \text { TSH } & \text { Thyroid stimulating hormone } \\ \text { DTC } & \text { Differentiated thyroid carcinomas } \\ \text { RAI } & \text { Radioactive iodine } \\ \text { Tg } & \text { Thyroglobulin } \\ \text { TgAb } & \text { Antithyroglobulin } \\ \text { WBS } & \text { Whole body scintigraphy } \\ \text { FDG } & \text { Fluorodeoxyglucose } \\ \text { PET } & \text { Positron emission tomography } \\ \text { Tc99m } & \text { Technetium-99 m }\end{array}$


TDC Thyroglossal duct cyst

PA Parathyroid adenoma

iPTH Intact parathyroid hormone

\section{Introduction}

Thyroid disorders are common and include many entities. They can be symptomatic, asymptomatic, diffuse, focal, neoplastic, or non-neoplastic processes. Neck ultrasound (US), with the prospect of proceeding to fine needle aspiration (FNA), is the first line of investigation; however, other options are available. Thyroid Uptake Scans using Tc-99 m or I-123 are typically reserved for specific clinical scenarios. Cross-sectional imaging including computed tomography (CT) and magnetic resonance imaging (MRI) detect incidental thyroid nodules (ITNs) and can be used in the evaluation of thyroid cancers and goiter $[1,2]$. The aim of this article is to provide a pictorial review of a broad spectrum of incidental and nonincidental thyroid findings on $\mathrm{CT}$ scans.

\section{CT scans of the thyroid: normal anatomy and imaging techniques}

The thyroid gland is a vascular, encapsulated structure made up of right and left lobes, which are connected at the midline by the isthmus. Each lobe is about $2 \mathrm{~cm}$ thick, $3 \mathrm{~cm}$ wide, and $5 \mathrm{~cm}$ long. The thyroid apex is located superiorly at the level of the mid-thyroid cartilage. The inferior margin of the gland is at the level of the fifth or sixth tracheal ring. The thyroid gland is encapsulated by the middle layer of deep cervical fascia and is part of the visceral space in the infrahyoid neck. It wraps around the trachea and is separated from the oesophagus by the tracheoesophageal groove on each side, which houses the recurrent laryngeal nerves. The thyroid has variable lymphatic drainage to the internal jugular chain, paratracheal region, mediastinum, and retropharyngeal area. It has homogeneous high attenuation values on a CT scan, as compared to adjacent muscles, due to its high iodine concentration. It shows avid iodine contrast enhancement due to its hypervascularity $[1,3]$.

Multi-detector volumetric acquisition from the skull base to the tracheal bifurcation is usually obtained. Multiplanar 2-mm axial, coronal, and sagittal images are typically available. Examination can be acquired with or without administration of intravenous (IV) iodinated contrast.

\section{Incidental thyroid findings on CT scan}

The thyroid gland can have variable CT scan findings, such as calcifications, single or multiple nodules, cysts, or diffuse enlargement.

Thyroid calcifications on a CT scan can be seen in both benign and malignant thyroid lesions [1]. Sonographic examination of the thyroid can differentiate between microcalcifications, which are highly associated with papillary thyroid carcinoma, and eggshell calcifications, which favour a benign process such as colloid cysts (Figs. 1 and 2) [4]. In a retrospective review of preoperative CT scan, $35 \%$ (135 of 383$)$ of the patients had detectable intrathyroidal calcifications. Among them, $48 \%$ had a histopathologically proven thyroid cancer. Calcified nodules had a significantly higher incidence of thyroid cancer and lymph node metastases. The incidence of thyroid cancer among nodules with different calcifications patterns were $79 \%$ of nodules with multiple punctate calcifications, $58 \%$ of nodules with a single punctate calcification, $21 \%$ of nodules with coarse calcification, and $22 \%$ of nodules with peripheral calcification. Most of the single calcified nodules were malignant [5]. However, this did not include patients with ITNs and the sample is skewed towards malignancy. Another study evaluated the presence of ITNs on CT scans and found that $12 \%$ of thyroid nodules were calcified, with no significant correlation between malignant or potentially malignant histology and punctate calcifications [6]. As a result, some researchers believe that calcification per se is not a suspicious CT sign, and have suggested that calcified thyroid nodules on CT scans should be treated the same as non-calcified nodules [7].

Thyroid cystic changes are variable, ranging from simple cysts with a thin wall to complex cysts with septations and solid components. An adenoma may undergo cystic degeneration. It is important to note that papillary carcinoma may mimic a benign-looking cyst. Simple serous cysts appear with fluid density on a CT scan, whereas a cyst with haemorrhage or high thyroglobulin content is iso-dense to muscle [1].

Thyroid nodules that are detected by an imaging study but have not been previously detected or suspected clinically are considered to be ITNs. ITNs are one of the most common incidental findings on neck imaging. ITNs are reported in up to $25 \%$ of chest CT scans, and in $16-18 \%$ of cervical region cross-sectional imaging, including CT and MRI scans. The rate of malignancy in the detected ITNs on CT and MRI scans varies from $0 \%$ to $11 \%[6,8-10]$. Incidentally detected thyroid carcinomas are more likely to be papillary thyroid carcinomas (PTCs) (Fig. 3). Incidentally detected cancers tend to be smaller in size and less likely to have distant metastasis, as compared to clinically suspected thyroid cancers [11]. 


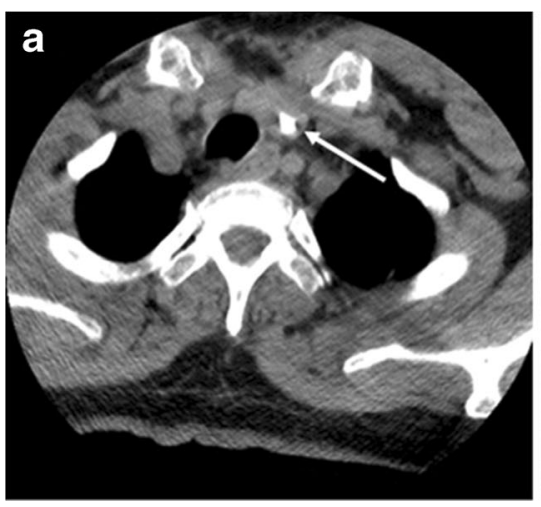

Fig. 1 An incidentally discovered colloid nodule with calcification, shown on CT scan of a 58-year-old female patient. a Non-enhanced axial CT scan of the neck demonstrates a coarse calcification at the left thyroid inferior pole. b Sagittal grey scale ultrasound of the thyroid demonstrates

On CT scans, a malignant lesion is suspected when the margins are ill-defined and there is extra-thyroid extension, lymph node involvement, or invasion of the surrounding structures. The absence of these features does not exclude malignant tumours, especially papillary, follicular, and medullary thyroid carcinomas (Fig. 3) [1]. Therefore, ultrasound is the modality of choice for thyroid lesion evaluation, due to its superior spatial resolution compared to CT examinations. Sonographic features of malignancy are micro-calcifications, acoustic shadowing, anti-parallel orientation, marked hypoechogenicity, irregular or microlobulated margins, and increased vascularity. CT scans lack the ability to detect these reliable sonographic signs of malignancy. Therefore, further management of ITNs, if required, usually begins with thyroid ultrasound and FNA should be considered according to the ultrasound findings [4, 12-15].

The American College of Radiology (ACR) flowchart and recommendations for ITNs detected by CT or MRI

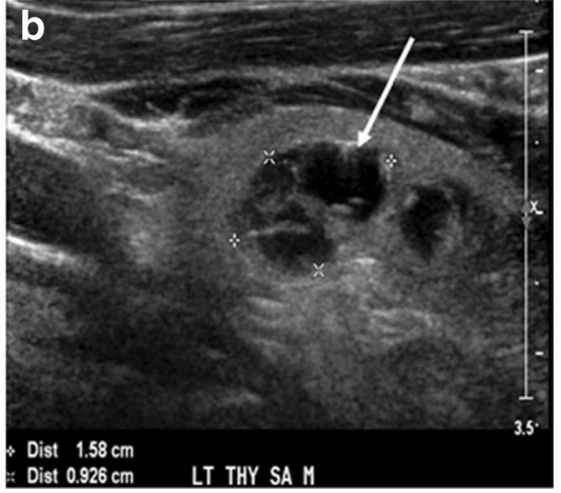

a heterogeneous nodule with predominant cystic component. Calcification was not seen in the ultrasound, probably due to its lower location in the superior mediastinum

offer general guidance and are not applicable to all patients. The recommendations are primarily based on the presence or absence of suspicious features, nodule size, patient's age, patient's life expectancy, and patient's comorbidities [11]. Suspicious features that can be detected on CT scans include signs of local invasion and abnormal lymph nodes. Abnormal lymph nodes may show cystic components, calcifications, and/or increased enhancement. Mere nodal enlargement is less specific for thyroid cancer metastasis; however, further evaluation should be considered if the ITN has ipsilateral jugulo-digastric lymph nodes $>1.5 \mathrm{~cm}$ on the short axis or $>1 \mathrm{~cm}$ for other groups. Cervical Level IV and VI lymphadenopathies raise a higher suspicion of thyroid carcinoma metastasis. Almost all patients with ITNs and suspicious imaging features should be evaluated with a neck ultrasound. Patients with comorbidities or limited life expectancy without suspicious features should not undergo further evaluation. Nevertheless, further workup might be

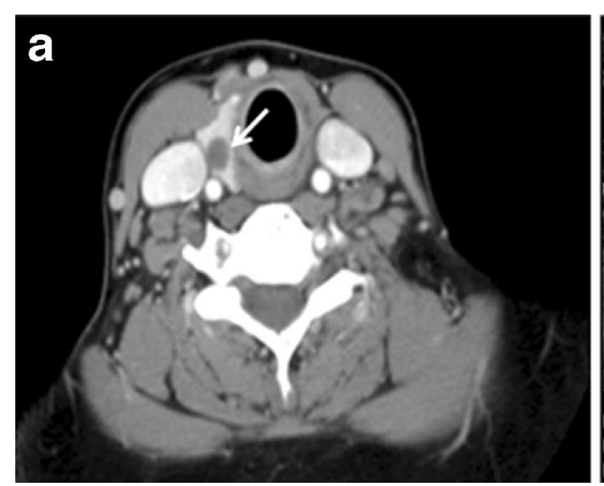

Fig. 2 A 51-year-old female patient post left hemi-thyroidectomy, with incidentally discovered right thyroid colloid nodule on CT scan. a Enhanced axial CT scan of the neck demonstrates a well-defined, hypodense right thyroid nodule (white arrow) with no internal calcifications or cervical lymphadenopathy. b Transverse greyscale

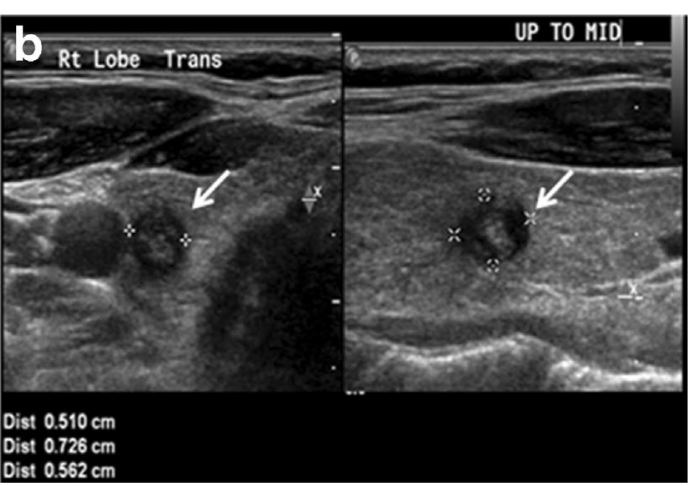

thyroid ultrasound demonstrates a well-defined, hypoechoic right thyroid lobe nodule with a central echogenicity including comet tail (ring down) artefacts (white arrow). No vascularity (not shown) or calcifications were detected 
Fig. 3 An incidental PTC in a 62-year-old male patient with lymphoma. a, b Enhanced axial CT scan and fused PET/CT scan of the neck demonstrate a welldefined, hypodense right thyroid nodule (white arrow) with high FDG uptake. The FDG-avid uptake in the left side (circle) is related to patient's known lymphoma, which resolved after treatment. c, d Transverse greyscale and sagittal colour Doppler ultrasound of the neck demonstrate a right thyroid irregular hypoechoic lesion with some micro-calcifications (white arrows) and increased vascularity
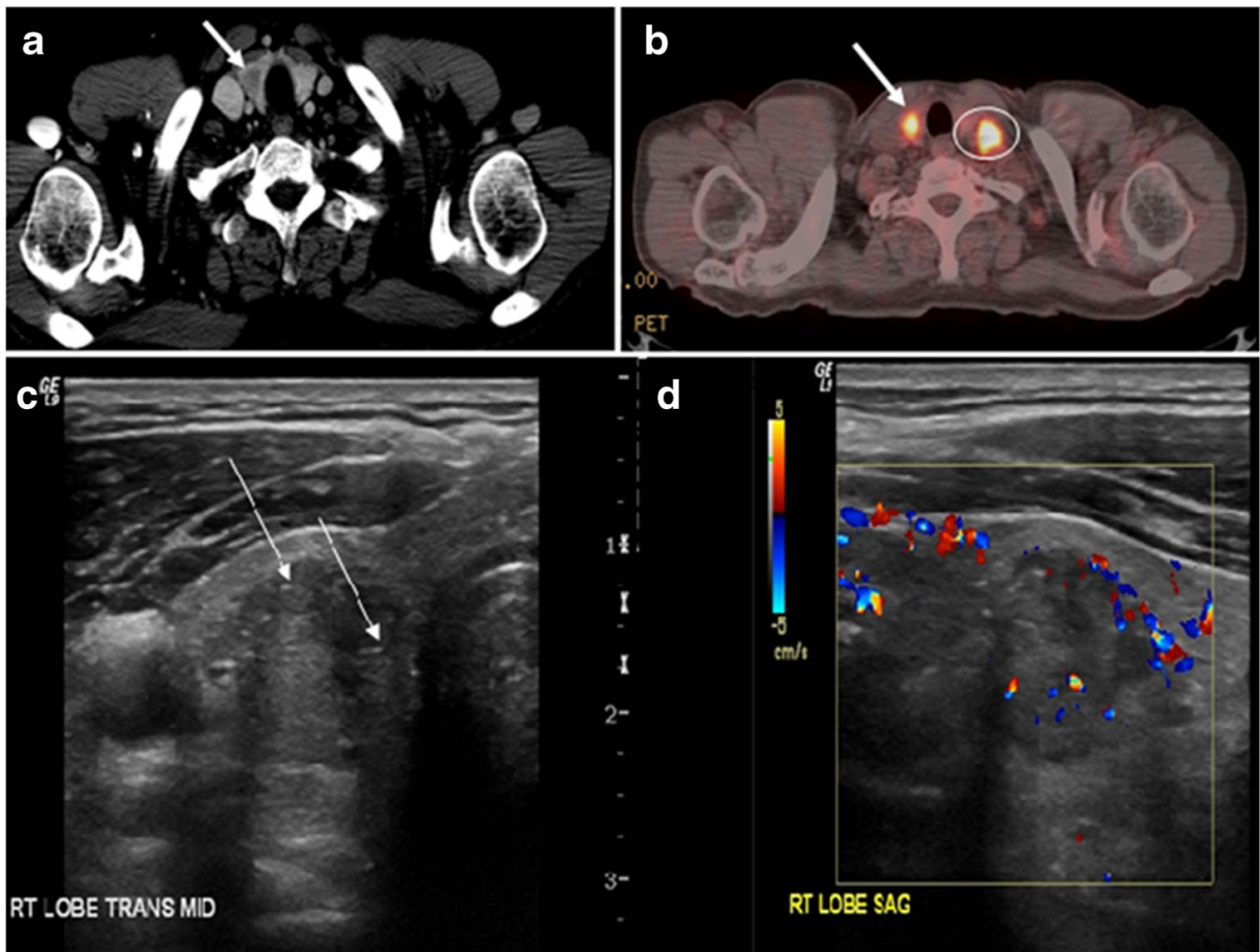

d

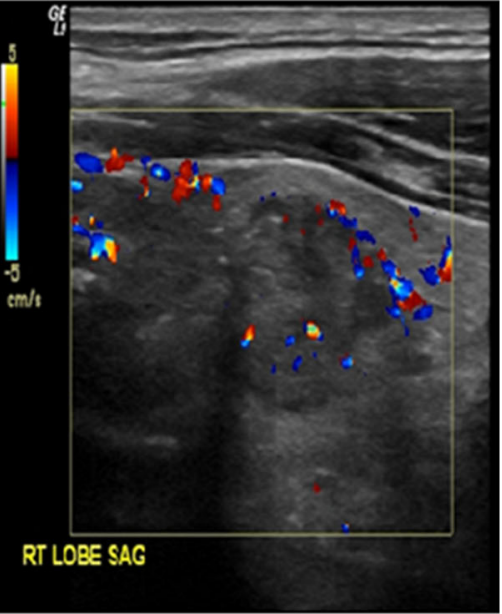

necessary for such individuals if it is clinically warranted, or specifically requested by the referring physician or the patient [11].

In patients with ITNs, it is important to inquire about pertinent historical factors predicting malignancy. These factors include history of childhood or adolescent head and neck or total body radiation exposure, and familial thyroid carcinoma or thyroid cancer syndrome. Syndromes associated with thyroid cancer include multiple endocrine neoplasia 2, familial adenomatous polyposis, Carney complex, Cowden's disease, and Werner syndrome/progeria. If a patient has a first-degree relative with such a syndrome, screening based on the various components of that syndrome is advised. Nevertheless, there are no guidelines specifically addressing ITNs detected on CT scans in patients at risk of thyroid cancer. Therefore, in the absence of suspicious features on the CT scan, other criteria such as nodule size on the CT scan, patient age, and levels of thyroid stimulating hormone (TSH) are important in guiding management in such a patient population [16].

Although the correlation between thyroid nodule size and malignancy risk is limited, nodule size affects prognosis in malignant nodules [13]. Small thyroid cancers (less than $2 \mathrm{~cm}$ ) tend to have an indolent course, with favourable prognosis even if not treated $[9,17]$. Less than $7 \%$ of the imaging-detected ITNs are seen in younger populations [6, 9, 18]. However, Shetty et al. [6] found a higher rate of malignancy in the ITNs detected on CT scans among patients younger than 35 years. Ito et al. [19] found a higher tumour progression risk among young patients $(<40$ years) with subclinical, low-risk PTCs who undergo observation rather than surgery. Therefore, nodule size and patient age should determine the need for workup in the general population without suspicious imaging features and with normal life expectancy. Further evaluation with ultrasound is required for patients less than 35 years old with nodules measuring more than $1 \mathrm{~cm}$ in the axial plane. The cutoff size for further evaluation is raised to $1.5 \mathrm{~cm}$ for patients more than 35 years old. This recommendation should be applied to the largest thyroid nodule in cases of multiple thyroid nodules. Incidentally discovered heterogeneous and enlarged thyroid glands should undergo dedicated ultrasound if the patient has no limited life expectancy or serious comorbidities [11].

\section{Thyroid cancers}

\section{Epidemiology}

Primary thyroid carcinomas include papillary, follicular, medullary, and anaplastic carcinomas. Lymphoma and metastasis of other primary malignancies to the thyroid gland represent a minority of thyroid carcinomas. Differentiated thyroid carcinomas (DTCs) originate from follicular epithelial cells and encompass PTCs and follicular thyroid carcinomas, including the Hurthle 
cell variant of follicular carcinoma. DTCs have an excellent prognosis and fortunately represent the majority of thyroid carcinomas. PTCs and follicular thyroid carcinomas represent $88 \%$ and $8 \%$, respectively, of all thyroid malignancies. Medullary thyroid carcinoma arises from neuroendocrine C-cells and has a good prognosis. Anaplastic carcinoma is an aggressive undifferentiated tumour that usually affects the elderly and tends to have a worse prognosis $[1,2,20,21]$.

\section{Role of imaging}

Surgery is the primary mode of treatment for DTCs. Post total thyroidectomy radioactive iodine (RAI) ablation is an option, especially in patients with distant metastasis, tumours larger than $4 \mathrm{~cm}$, or extra-thyroidal disease extension. Ultrasound examination is usually adequate in evaluating primary tumours and cervical lymph nodes. Preoperative cross-sectional imaging with CT or MRI is indicated if there is a concern for local invasion that may alter the patient's staging as well as surgical approach (Figs. 4, 5 and 6) [2]. Some thyroid primaries may be small, diffuse, or multifocal and therefore may be occult on imaging (Fig. 4) [6].

In patients with known thyroid malignancies, a nonenhanced exam is preferred due to the possible undesired interference of free iodide contrast medium with thyroid iodide I-131 uptake for 6-8 weeks or more [22, 23]. This would adversely affect the management of these patients by delaying diagnostic thyroid scintigraphy and radioiodine ablation in patients with DTCs for 2-6 months.

The radiologist must evaluate the central structures draping the thyroid gland including the trachea, oesophagus, larynx, and pharynx, as well as the recurrent laryngeal nerve. Invasion is suspected if the thyroid mass abuts the airway or oesophagus for more than 180 degrees. Luminal deformity, mucosal thickening, and mucosal focal irregularity are more specific indicators of invasion.
Obliteration of the fat planes of the tracheoesophageal groove in three axial images and signs of vocal cord paralysis are indicative of recurrent laryngeal nerve invasion. Invasion of these central structures meets the criteria for T4a disease (Figs. 5 and 6) [2].

Arterial invasion constitutes $\mathrm{T} 4 \mathrm{~b}$ disease, which may preclude curative surgery. More than 180 degrees of arterial encasement is suggestive of invasion, however, arterial deformity or narrowing is much more suspicious for invasion [2]. The carotid artery is the most commonly involved artery; however, the mediastinal vessels should also be examined. Encasement of the carotid artery or mediastinal vessels for more than 270 degrees is unlikely to be resectable [24]. On the other hand, occlusion or effacement of the internal jugular vein can occur without invasion and does not influence surgical resectability or staging. Asymmetry of the strap muscle and the tumour abutting its external surface are signs of invasion. However, invasion of the pre-vertebral musculature is more challenging, as a large lesion can compress the muscle without invasion (Figs. 5 and 6) [2, 25].

Finally, the possibility of metastatic disease should be excluded. PTCs and medullary thyroid carcinomas tend to metastasize to regional lymph nodes. According to the AJCC/UICC TNM staging system, the nodal stage is classified by site: N1a indicates level VI nodal involvement, including paratracheal nodes; N1b indicates unilateral or bilateral lateral cervical nodal disease or superior mediastinal nodal disease (Figs. 4, 5 and 6) [2, 25].

The incidence of hematogenous spread of follicular carcinomas is $21-33 \%$ and that of PTCs is $2-14 \%$ [26, 27]. In medullary thyroid cancer and anaplastic thyroid cancer, distant metastasis was reported in $25 \%$ and $40 \%$ of patients, respectively. Distant metastases from DTCs tend to have a more favourable prognosis. Distant metastatic disease may appear years after the initial presentation. Therefore, imaging for distant metastases is usually done pre-operatively for anaplastic thyroid cancer and post-operatively for DTCs. DTC distant metastases sites include the lung ( $50 \%$ ), bone (25\%), lung and bone (20\%), followed by other sites $(5 \%)$ [28-30].

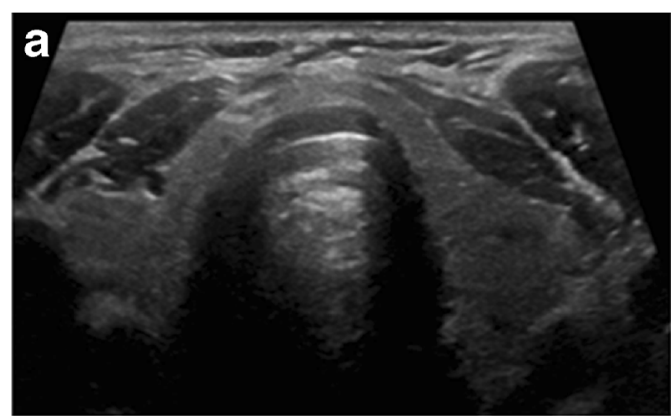

Fig. 4 A 45-year-old male patient presented with anterior mediastinal metastatic PTC lesions and occult primary on imaging. Histopathology examination of the resected thyroid gland revealed micro-foci of PTC; the largest, in the isthmus, measured $4 \mathrm{~mm}$. a Transverse greyscale ultrasound of the thyroid demonstrates homogenous gland with normal

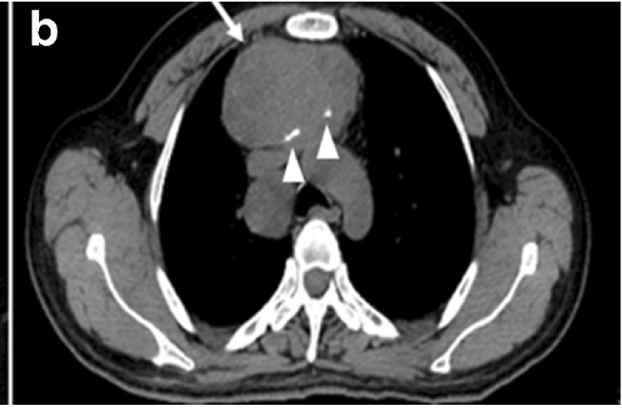

echogenicity and size. No focal lesion or micro-calcifications. b Nonenhanced CT scan obtained as part of PET/CT examination shows a heterogeneous, large, relatively dense anterior mediastinal mass (white arrow) with peripheral calcification (arrowheads). Thyroid gland has normal CT appearance with no abnormal FDG uptake (not shown) 

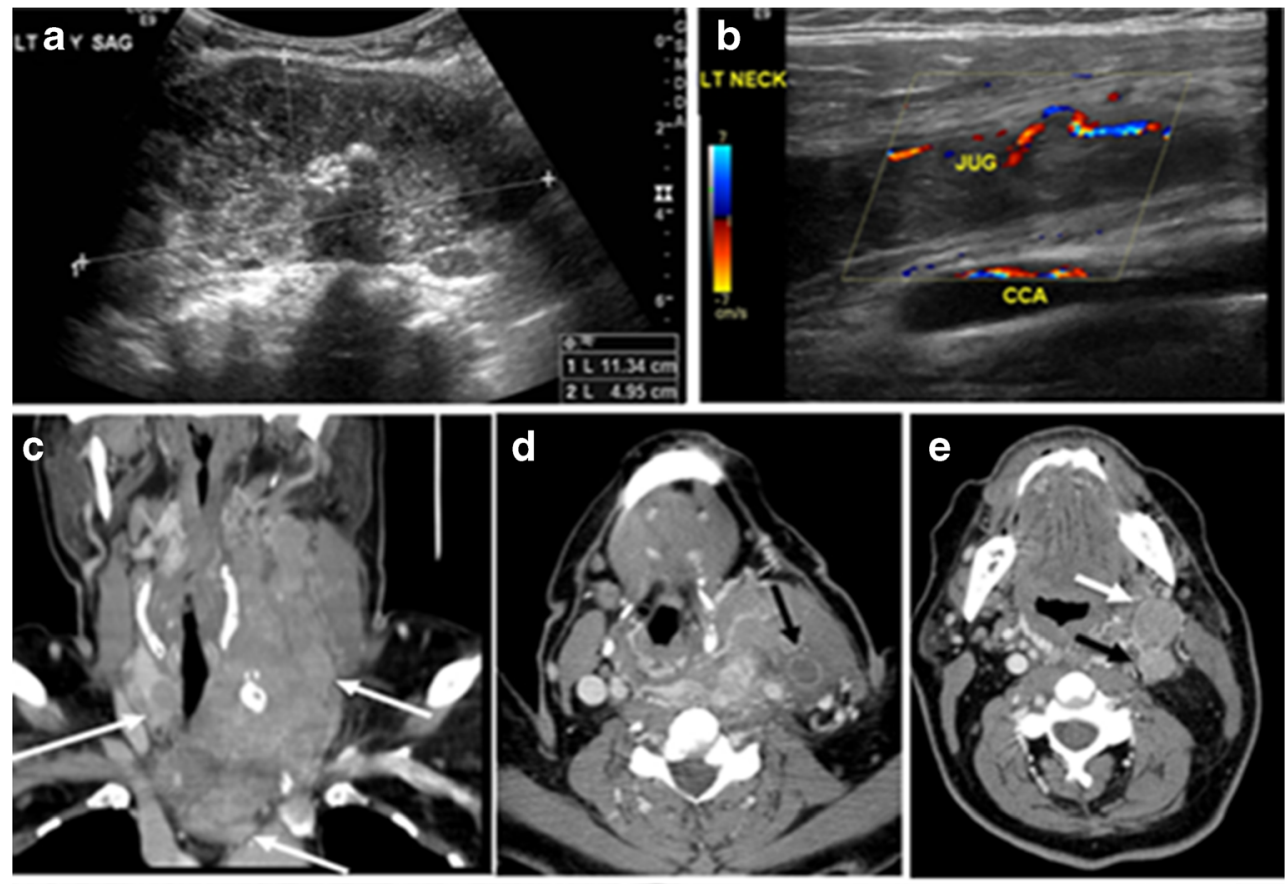

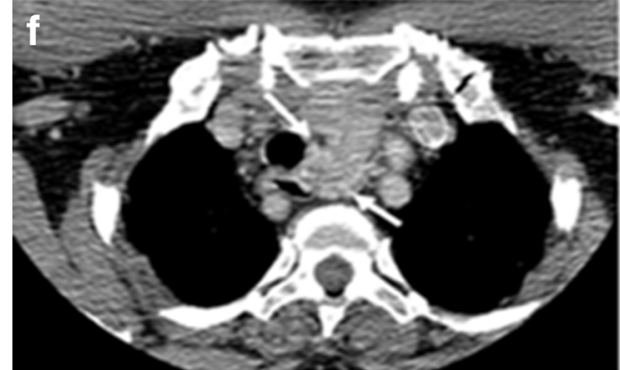

Fig. 5 A poorly differentiated invasive left thyroid mass in a 58-year-old female patient. a Sagittal greyscale neck ultrasound shows a large hypoechoic lesion with macro-calcification and micro-calcification. b Sagittal colour Doppler ultrasound shows left internal jugular vein filling defect with detected internal vascularity suggestive of tumour thrombus. c Enhanced axial and coronal CT scans of the neck show heterogeneously enhancing large lesion replacing the left thyroid lobe and extending to the isthmus and the medial aspect of the right thyroid lobe (white arrow). The mass and the conglomerate lymph nodes measure

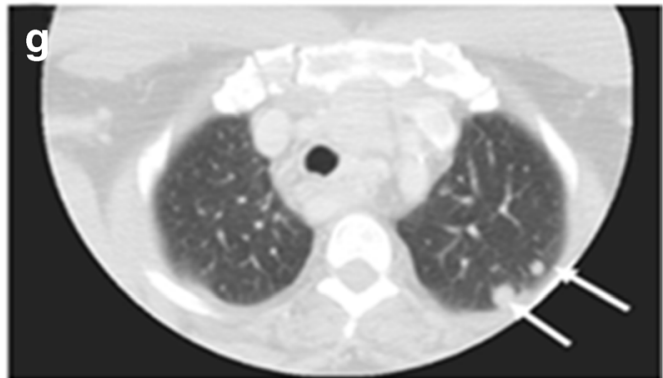

$12.5 \times 7 \times 5.8 \mathrm{~cm}$ (white arrows). d, e Axial enhanced CT scans show enlarged left cervical nodes (white arrow) and left internal jugular vein (IJV) thrombus (black arrows). Note the IJV distention and central enhancing portion in the upper cut (black arrow in e) concerning the tumour thrombus. f, g Enhanced axial CT scan of the upper chest demonstrate a mass extension into the retrosternal area, left tracheoesophageal groove, and posterior to the trachea (white arrows in f). There are multiple bilateral pulmonary nodules (white arrows in $\mathbf{g}$ )
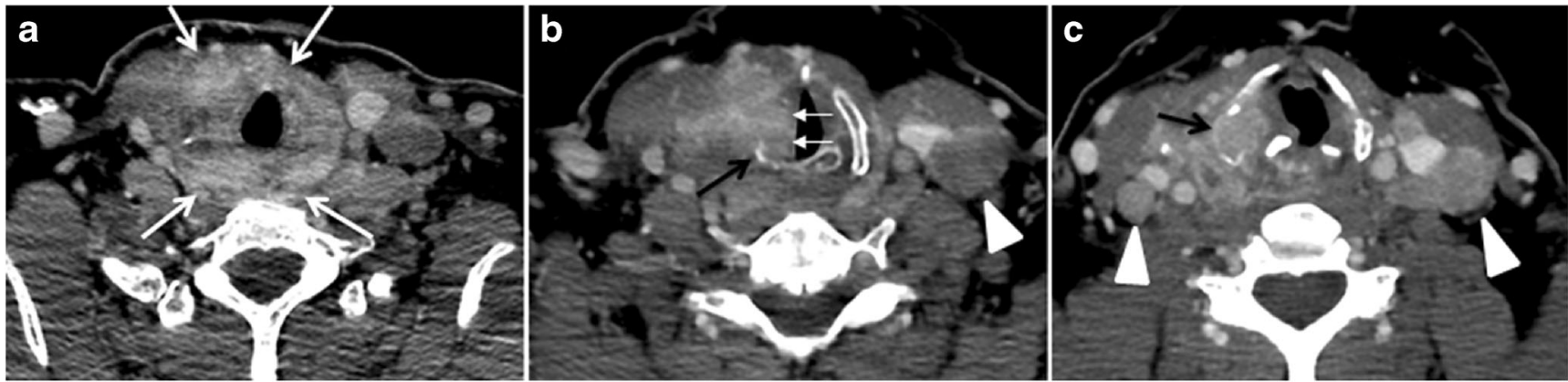

Fig. 6 A 61-year-old female patient with locally aggressive PTC. a Enhanced axial CT scan of the neck demonstrates a heterogeneous infiltrative thyroid mass. This mass diffusely involves the entire gland and circumferentially encases the trachea with involvement of bilateral tracheoesophageal grooves (white arrows). b, c Additional axial cranial images show right cricoid cartilage destruction (black arrows in b), right thyroid cartilage destruction (black arrow in c), right vocal cord paralysis (white arrows in $\mathbf{b}$ ), and bilateral cervical lymphadenopathy (arrowheads) 


\section{Recurrence}

The thyroid cancer recurrence rate is reported to range from $7 \%$ to $14 \%[31,32]$. Recurrence is usually detected within the first decade after initial disease diagnosis. Large lymph node metastasis is considered the strongest predictor for thyroid cancer recurrence [31]. Post-treatment surveillance for recurrent disease depends on cancer type and staging. Patients with DTC are usually treated with total thyroidectomy and RAI ablation. Patients should have baseline neck US evaluation at 6-12 months after the RAI ablation and then periodically, depending on the patient's risk for recurrent disease and thyroglobulin ( $\mathrm{Tg}$ ) status. After the first post-operative RAI ablation, further RAI imaging is not necessary if the patient has normal neck US, undetectable Tg level under TSH stimulation, and negative antithyroglobulin (TgAb). Annual neck US with or without FNA, along with measurement of serum $\mathrm{Tg}$ and serum $\mathrm{TgAb}$, is usually sufficient for post-treatment surveillance in those patients. Moreover, annual US is appropriate in patients with medullary cancer and normal calcitonin levels $[2,16,33]$.

The likelihood of positive anatomic imaging is greater when serum $\mathrm{Tg}$ is $>10 \mathrm{ng} / \mathrm{mL}$ [16]. A diagnostic CT scan adds additional value to neck US in detecting central compartment macro-metastases in the mediastinum and retro-tracheal area [34-36]. According to the recent American Thyroid Association guidelines [16], an upper chest and neck CT scan with IV contrast should be obtained when: 1) neck US is inadequate in visualizing possible local nodal disease (high Tg, negative neck US, and RAI imaging); 2) US is not able to delineate the disease completely, as in the case of bulky recurrent nodal disease; or 3 ) evaluation of possible recurrent invasive disease is needed (Figs. 7, 8 and 9). CT scans are also the most sensitive diagnostic tool for the detection of pulmonary micro-metastases. Many of the neck US features that are considered as suggestive signs of disease recurrence are also applicable to CT examination. These signs might include sizable rounded nodules in the thyroid bed, fine calcifications, or cystic change [37].

In cases of elevated thyroglobulin with negative neck US and iodine whole body scintigraphy (WBS), fluorodeoxyglucose (FDG) positron emission tomography (PET) is the next modality of choice. Dedifferentiated thyroid carcinoma usually has avid FDG-PET uptake and a negative radioiodine scan, typically does not respond to RAI therapy, and has a poorer prognosis [2]. There is not yet consensus in the research literature on whether cross-sectional imaging (CT or MRI) or an 18FDG-PET/CT scan should be performed as the first-line imaging modality for such patients. Enhanced CT scan was thought to be more sensitive for detection of lymph node metastases. Nonetheless, scans using modern PET/CT equipment are as reliable as a proper routine staging $\mathrm{CT}$ scan. Many lesions can be found on 18FDG-PET/CT scanning despite the lack of IV contrast injection. However, differentiation between local recurrence versus lymph node metastases and detection of direct involvement of the aerodigestive axis or vascular structures are not technically possible in the absence of IV contrast administration. For these reasons, 18FDG-PET/ CT utilizing contrast administration should be considered for most patients with extensive disease [16, 38-40].

\section{Metastasis to the thyroid}

Metastasis to the thyroid is rare and represents $5.5 \%$ of biopsied thyroid malignancies. It is commonly found with cancers originating from the breast, renal cell, lung, melanoma, and colon. Direct invasion from adjacent structures such as the pharynx, larynx, trachea, or oesophagus has been reported (Fig. 10). Metastatic disease has a non-specific appearance [1, 2, 20, 21].

Presence of ITNs in patients with another known malignancy is a common clinical problem with controversial management guidelines. Wilhelm et al. [41] followed 41 patients

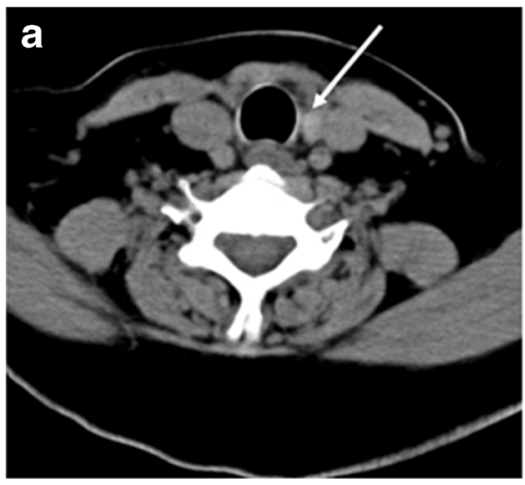

Fig. 7 A 51-year-old female patient post total thyroidectomy for PTC with elevated thyroglobulin measurement. a Axial non-enhanced CT scan of the neck at the level of the thyroid bed demonstrates a well-defined, rounded, homogenously dense soft tissue situated between the trachea and left internal jugular vein (white arrow). b Transverse ultrasound

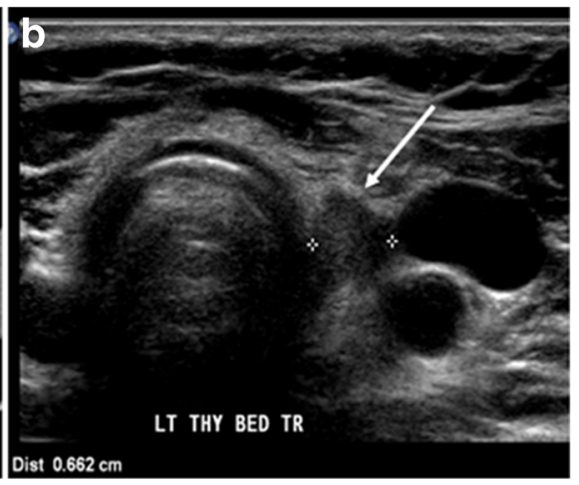

image of the neck demonstrates a well-defined, homogenous, hypoechoic soft tissue nodule measuring $6 \mathrm{~mm}$ (white arrow) with no detected micro-calcifications. Biopsy showed a predominantly residual normal thyroid tissue with micro-foci of PTC 

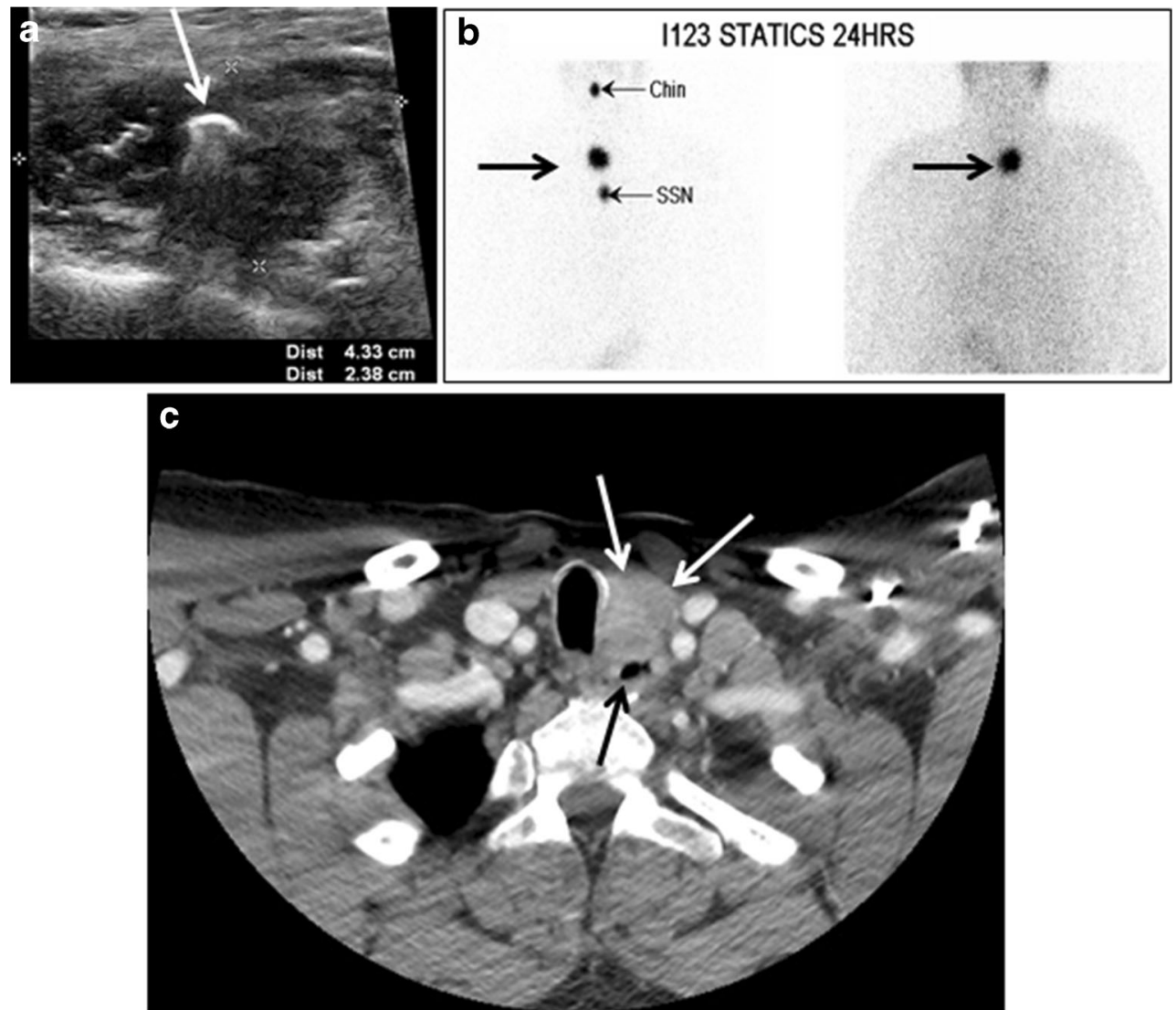

Fig. 8 A 48-year-old male patient post total thyroidectomy with PTC recurrence. a Transverse greyscale ultrasound of the neck demonstrates a left thyroid bed heterogeneous, predominantly hypoechoic irregular lesion with calcifications (white arrow). b A spot image of iodine 123 total body scan of the neck demonstrate a focus of abnormal radiotracer uptake at the left thyroid bed (Black arrows) between the annotated markers. c Enhanced axial CT scan of the neck demonstrates an enhancing large left thyroid bed mass (white arrow) with no calcifications. The lesion exerts a mass effect on the oesophagus (black arrow) and is inseparable from the trachea with a known extra-thyroid malignancy and ITNs; 35 of them met the criterion for biopsy (nodule $\geq 1 \mathrm{~cm}$ ). Pathology revealed four papillary thyroid cancers and five micropapillary thyroid cancers. Only two metastatic cancers were detected. Clinical history (history of radiation, age, endocrine syndromes), TSH, nodule size, and sonographic features are important to determine which nodule(s) should be followed or biopsied. However, existing guidelines do not specifically address how to approach ITNs detected on CT scans in such a specific patient population [41].

\section{Thyroid lymphoma}

Thyroid lymphoma represents about $5 \%$ of thyroid malignancies. Non-Hodgkin's lymphoma is the most common type and can be secondary to generalized lymphoma or a primary tumour. Primary thyroid lymphoma usually pre-exists with Hashimoto's thyroiditis. On CT scans with and without contrast, lymphomas tend to have low attenuation values. Thyroid lymphomas have variable appearance and mostly manifest as a solitary mass $(80 \%)$. They may also manifest as multiple nodules (15\% to $20 \%$ ) or as a bulky mass replacing the entire gland with extra-thyroid extension (Figs. 11 and 12). The presence of cervical lymphadenopathy supports such a diagnosis. Although it is uncommon, tumour necrosis has been reported $[1,21]$.

\section{Goiter}

A goiter is an abnormal thyroid gland proliferation that manifests as multi-nodular, uni-nodular, or non-nodular diffuse glandular enlargement. A goiter is formed of solid matrix, colloid cysts, blood products, calcification, and fibrosis, and this heterogeneity may lead to variable appearances on a CT scan (Figs. 13, 14 and 15) [2]. US is more sensitive in evaluating thyroid nodules within a goiter; however, a symptomatic goiter may require surgical treatment with total thyroidectomy [42], and in this case CT plays an additional role in preoperative evaluation. Specific aspects for examination on a CT 

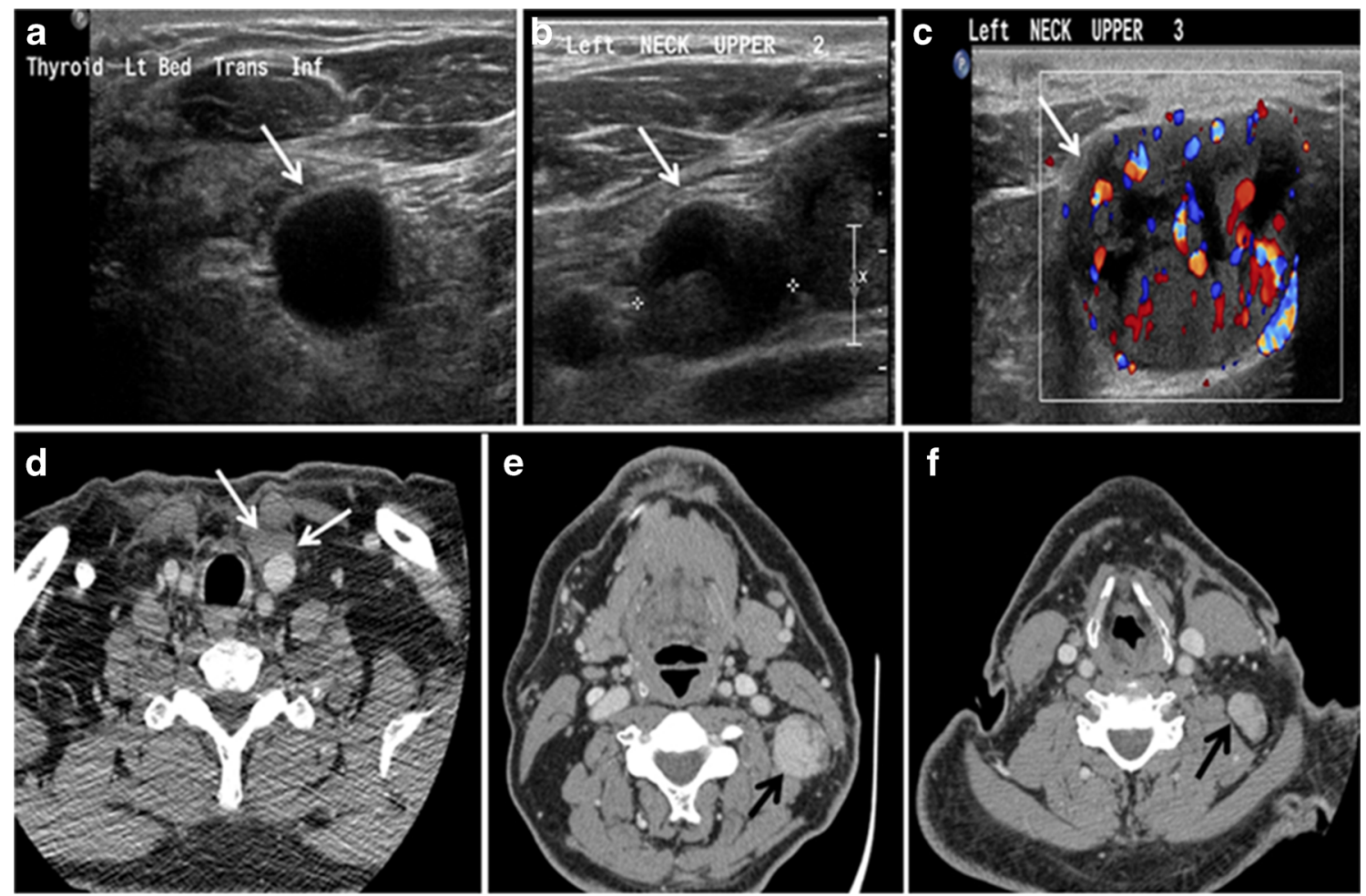

Fig. 9 A 58-year-old male patient with persistence PTC at thyroid bed with hypervascular nodal metastasis. a-c Transverse greyscale and colour Doppler neck ultrasound demonstrate hypoehoic soft tissue in the left thyroid bed (white arrow in a). There are a heterogeneous enlarged lymph nodes at level 2 and 3 with markedly increased vascularity

(white arrow in $\mathbf{b}$ and $\mathbf{c}$ ). $\mathbf{d}-\mathbf{f}$ Enhanced axial CT images of the neck demonstrate a $2.7 \times 1.4 \mathrm{~cm}$ hypodense soft tissue lesion anterior to the left carotid sheath (white arrow). There are left-sided enhancing abnormal and enlarged lymph nodes at cervical level 2 and 3 (black arrows)

scan during the preoperative evaluation for goiter include extension, mass effect, and suspicious features of malignancy [2, 43].

Malignancy can coexist within the goiter and a CT scan may give a clue if there are abnormal cervical lymph nodes and/or signs of invasion. Retrosternal extension (Fig. 15) could affect the surgical approach, as a lower extent may require a partial or total sternotomy to facilitate complete resection [43]. Therefore, the distance of the retrosternal extent from the sternal notch should be measured on a sagittal image.

The interpreting radiologist should describe the mass effect, detailing its degree and direction of displacement of central structures, including the trachea, oesophagus, larynx, and pharynx. Attention should be directed to the upper extent of the goiter and structures immediately surrounding the thyroid
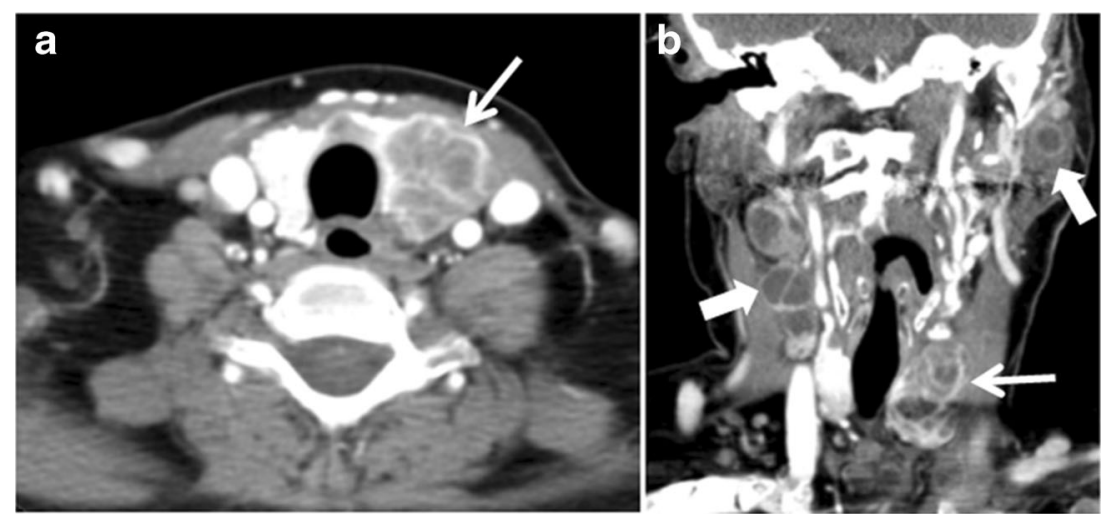

Fig. 10 Metastatic squamous cell carcinoma of unknown origin in a 42-year-old female patient. a, b Axial and coronal enhanced neck CT scan demonstrates infiltrative hypodense left thyroid lobe lesions (white arrows). There are multiple necrotic cervical nodal metastases (white block arrows) 


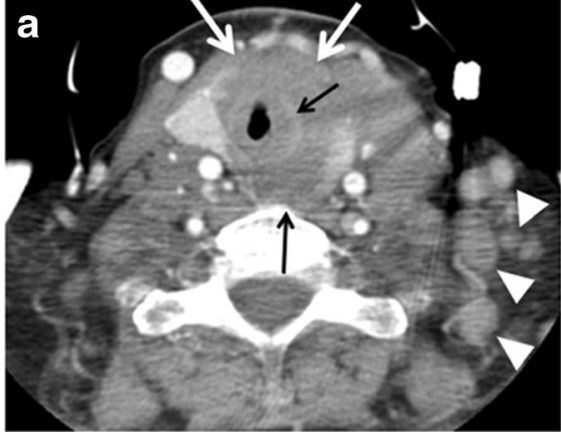

Fig. 11 Thyroid non-Hodgkin's large B-cell lymphoma in a 66-year-old female patient. a Axial enhanced neck CT scan demonstrates left thyroid lobe and isthmus homogeneously hypodense and minimally enhancing mass (white arrows). This lesion invades the prevertebral muscles (black

gland, including the neuro-vascular structures, retropharyngeal space, and pre-vertebral space. The reporting radiologist should evaluate the vocal cords for symmetry and signs of vocal cord palsy $[2,43]$.

\section{Inflammatory lesions}

Inflammatory thyroid disorders include acute infectious thyroiditis, Hashimoto's thyroiditis, Riedel's thyroiditis, and granulomatous thyroiditis (de Quervain's). Hashimoto's thyroiditis is associated with an increased risk of lymphoma and papillary thyroid carcinoma. The CT scan findings of thyroiditis are nonspecific and variable (Figs. 14, 15 and 16) [1]. The thyroid gland has a very high iodine concentration, resulting in high CT attenuation (80-100 Hounsfield Units). The presence of thyroiditis can be suggested by a diffusely enlarged and hypo-attenuating (around 45 Hounsfield Units) thyroid gland. This is probably due to follicular cell destruction and reduced thyroid iodine concentration. Marked homogenous enhancement is typically expected. Therefore, moderate thyroid enhancement in a case of thyroiditis suggests a diffuse inflammatory process. It is essential to clinically correlate this with a thyroid function test and serum autoantibody levels [1, 44].

\section{Ectopic tissue/gland}

During embryogenesis, the bi-lobed thyroid migrates inferiorly from the foramen cecum of the tongue to the lower neck. Initially, the thyroid primordium passes anterior to the primordial hyoid bone, before it loops posteriorly and inferiorly to the hyoid bone. Then it continues its descent into the infra-hyoid portion of the neck, anterior to the trachea, thyroid cartilage, and thyroid membrane. Any thyroid residual along the descent course may lead to the development of ectopic thyroid glands. Thyroid

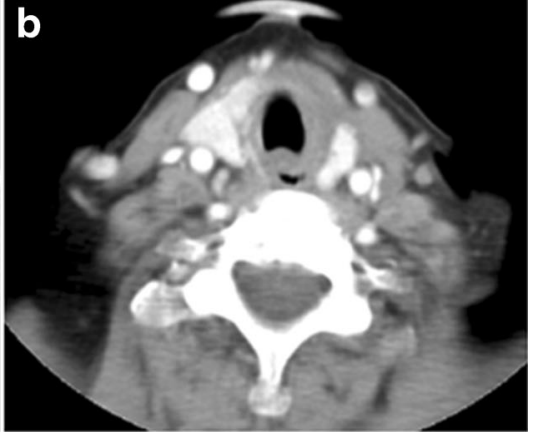

arrows). Note the multiple enlarged level V lymph nodes (white arrowheads). b Post-treatment image shows significant reduction in size and mass effect of the left thyroid infiltrative mass, with almost complete resolution of the left cervical lymphadenopathy

carcinomas, thyroiditis, and goiter may develop within any ectopic thyroid tissue [1, 45-47].

Thyroid scanning with technetium-99 $\mathrm{m}$ (Tc99m) plays an important role in detecting orthotopic and ectopic thyroid tissue. Both CT scans and US can help detect ectopic tissue when a lesion demonstrates imaging and enhancement characteristics of thyroid tissue. The absence of normally sited thyroid gland in US and CT scans also supports the diagnosis. In addition, US can guide FNA for cytological confirmation of a thyroid lesion [46]. Ectopic thyroid tissue appears as a well-circumscribed, homogeneous, highly attenuating mass relative to adjacent muscles. Normally, it enhances avidly following the administration of iodinated contrast $[1,45,47]$.

Ectopic thyroid tissue may be detected in the tongue near the foramen cecum $(90 \%)$ and along the midline between the thyroid isthmus and posterior tongue, lateral neck, mediastinum, and oral cavity. The most frequent location is the base of the tongue (Figs. 16, 17 and 18). In $70 \%$ of cases, the ectopic thyroid is the

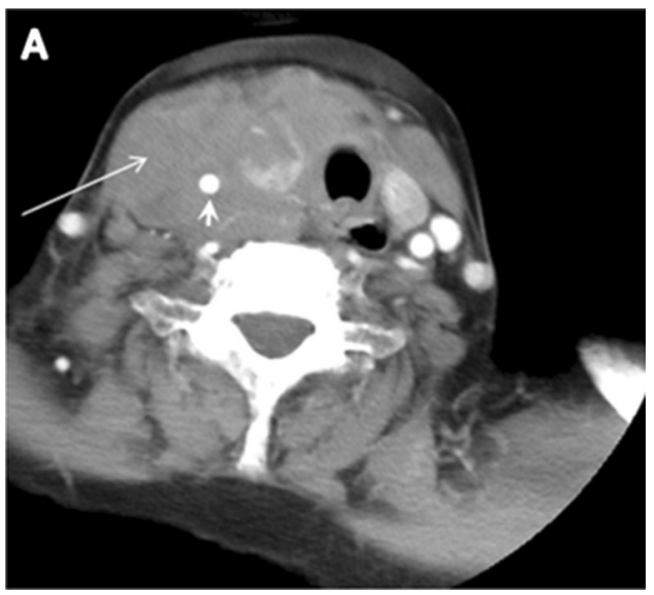

Fig. 12 Diffuse thyroid large B-cell lymphoma in 79-year-old female patient. a Axial enhanced neck CT scan demonstrates a homogeneously hypodense and minimally enhancing large right thyroid solid mass (long white arrow) extending into the thyroid isthmus. It is encasing the right carotid artery (short white arrow) and displacing of the trachea and oesophagus to the left side 


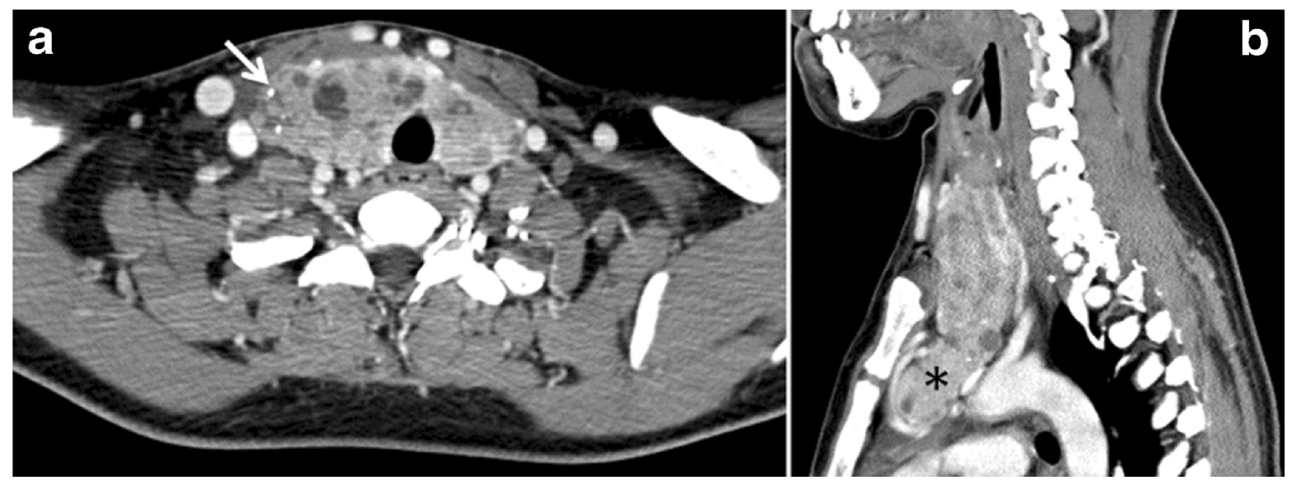

Fig. 13 A 27-year-old female patient known to have goiter. a, b Axial and sagittal enhanced CT scan images of the neck demonstrate a heterogeneously enhancing, enlarged thyroid gland with scattered

only functional thyroid tissue present in the body (Fig. 18) $[1,45$, 46].

Ectopic thyroid tissue lateral to the orthotopic midline location is rare $[46,48,49]$. The exact anatomical definition of this rare entity is debated in the literature $[48,50]$. To avoid confusion, some authors define a lateral neck ectopic thyroid as any thyroid tissue superficial to the strap muscles with no midline continuity calcifications (white arrow), cystic changes, and substantial retro-sternal extension (black asterisks). No lymphadenopathy or substantial airway narrowing
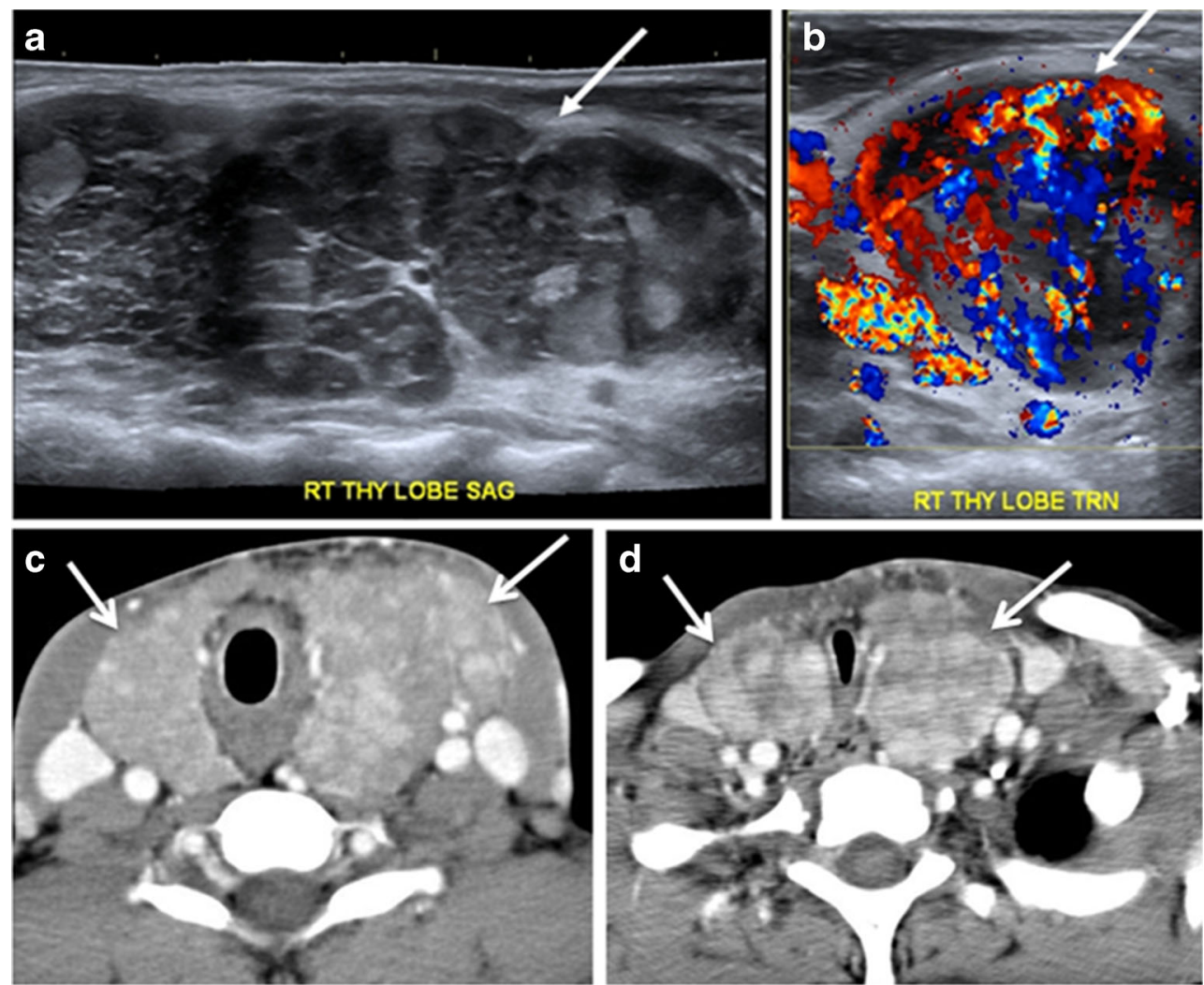

Fig. 14 A 19-year-old male patient known to have multi-nodular goiter and FNA, showing underlying Hashimoto's thyroiditis. a, b Sagittal and transverse greyscale and colour Doppler ultrasound of the neck demonstrate a hypoechoic enlarged right thyroid lobe with small
[45]. The majority of lateral thyroid ectopia cases have been reported as lesions closely related to the strap muscles [48, 49]. There are few reported cases of ectopic lateral thyroid tissue in the submandibular region [46, 51], jugulodigastric region [52], or within the parotid gland substance (Fig. 17) [53].

The origin of lateral ectopic thyroid tissue is not fully understood. Although this is controversial, some authors suggest that it 


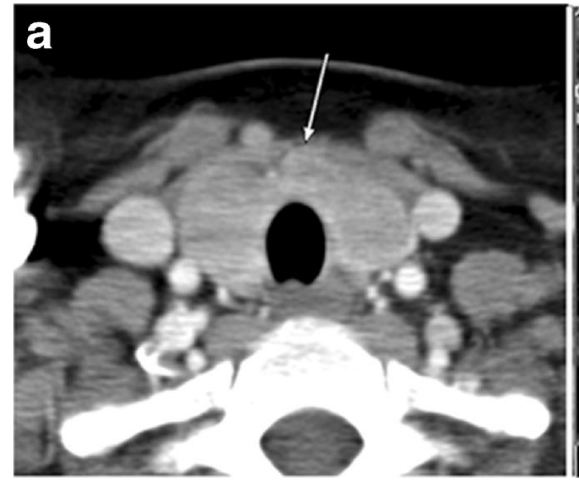

Fig. 15 A 33-year-old female patient who presented with neck swelling and pain and was later diagnosed with Hashimoto's thyroiditis. a Axial enhanced CT scan of the neck demonstrates minimal diffuse enlargement

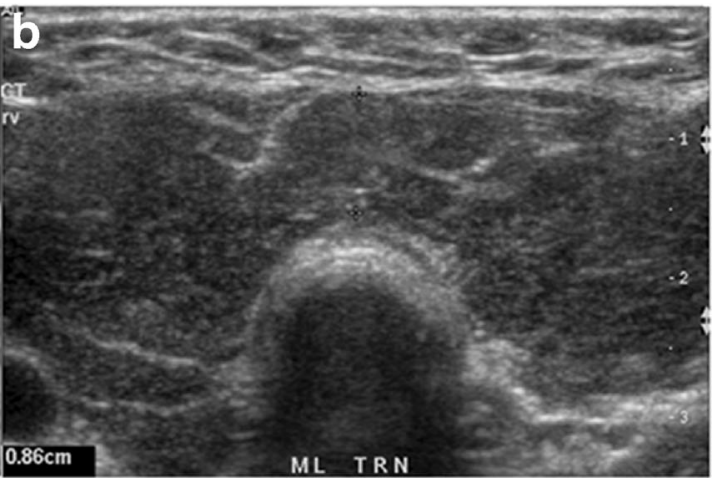

of the thyroid gland, especially the isthmus (white arrow). b Transverse greyscale ultrasound of the neck demonstrates heterogeneously enlarged thyroid and thickened isthmus, measuring $8.6 \mathrm{~mm}$ might have originated from lateral thyroid anlagen (ultimobranchial bodies) that failed to fuse with the median anlage during caudal migration [46, 48].

A thyroglossal duct cyst (TDC) is a duct remnant between the foramen cecum and thyroid isthmus. Most TDCs are located below the hyoid bone and in the midline. The more caudal the cyst, the more likely it will be off midline within $2 \mathrm{~cm}$ (Fig. 19 and 20). On a CT scan, a TDC appears as a wellcircumscribed area of fluid attenuation with thin walls. The cyst wall can become thick with an enhancing rim indicative of current or previous infection. These cysts maybe complicated by haemorrhage, infection, or malignancy. Therefore, their US and CT scan appearance may vary based on their content. Nodular enhancement within a TDC should initiate further workup to exclude malignancy (Fig. 21) [1, 45]. USguided FNA of these suspicious nodular areas is considered an appropriate next diagnostic step, taking into consideration the high rate of false negative results [54].

\section{Intra-thyroid parathyroid adenoma}

Parathyroid adenoma (PA) is the most common cause of primary hyperparathyroidism. Ectopic parathyroid adenoma is rare. The third and fourth pharyngeal pouches represent the embryological origin of the parathyroid tissues, and ectopic parathyroid adenoma can ultimately develop anywhere along their migration course. In a large retrospective study of

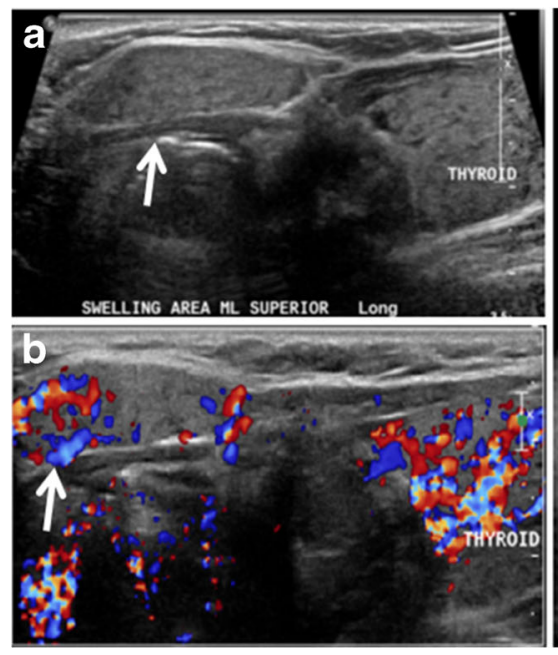

Fig. 16 Midline ectopic thyroid with Hashimoto's thyroiditis in a 49year-old female patient. a Transverse greyscale ultrasound shows a $1.6 \times 0.8 \mathrm{~cm}$ solid, well-defined, heterogeneous area (white arrow) in the midline, superior to the thyroid gland. It is iso-echogenic to the thyroid gland with no definite connection to the thyroid gland. b

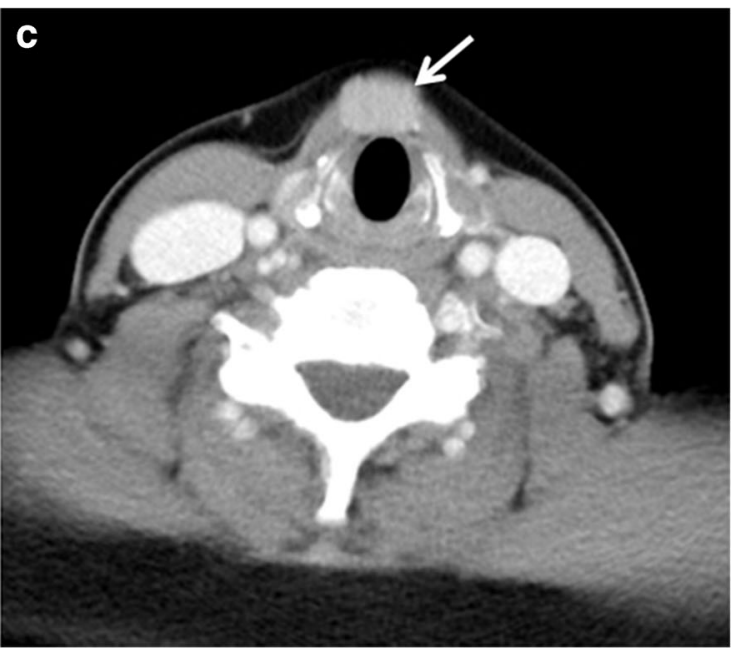

Transverse colour Doppler ultrasound shows significant increase in vascularity. c Axial enhanced neck CT scan at the level of thyroid cartilage demonstrates midline infrahyoid hyperdense soft tissue mass (white arrow) embedded within the strap muscle 

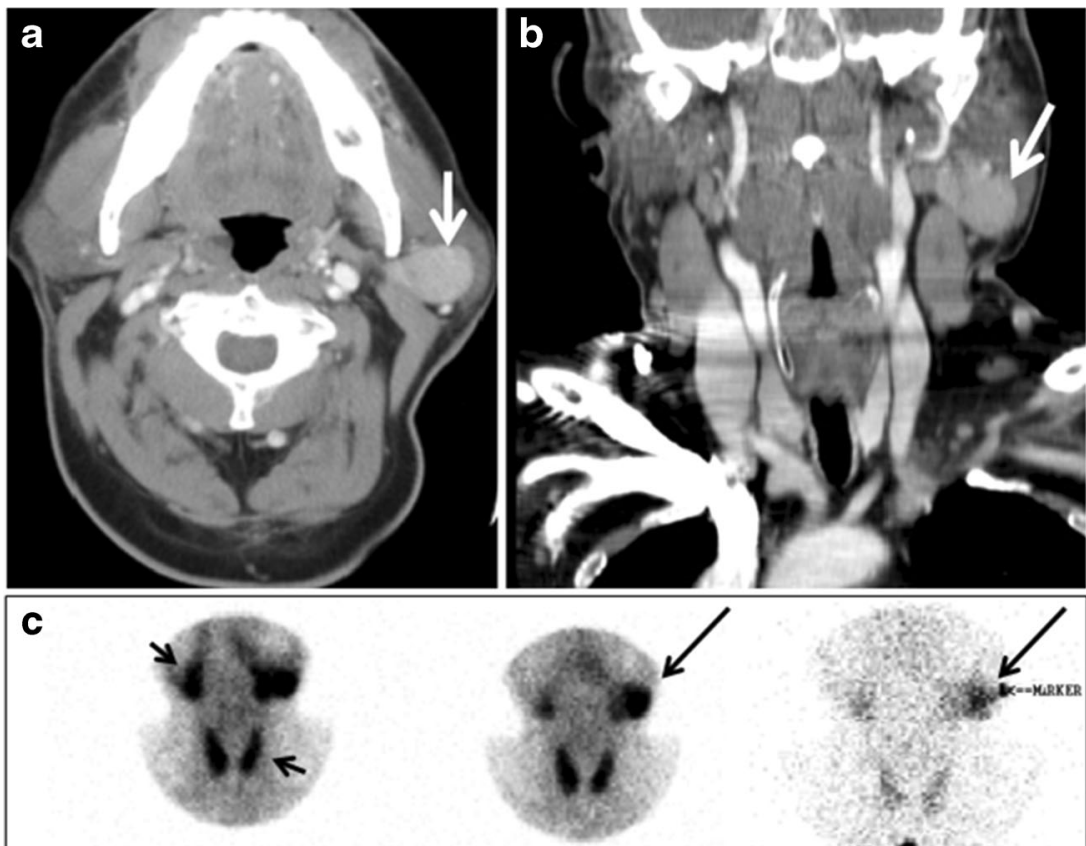

PRE LEMON

ANT Neck 20 MIN

POST LEMON

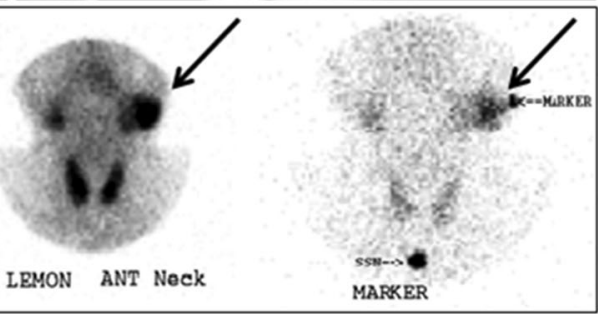

Fig. 17 Ectopic thyroid on the left parotid gland with a palpable left parotid mass in a 69-year-old male patient. a, b Axial and coronal enhanced neck CT scan demonstrates well-defined homogeneous enhancing mass (white arrows) within the left parotid gland with preserved surrounding fat planes. It also shows a normal thyroid in normal position in the lower neck. c Image taken 20 minutes after

patients with primary hyperparathyroidism, PA was detected in the intra-thyroid location in $0.7 \%$ of cases. In another retrospective analysis of 202 patients with ectopic PA, intrathyroidal location was found in $18 \%$ of the cases. Intrathyroid parathyroid adenomas mimic thyroid nodules in CT scans and may even show uptake on a thyroid iodine scan.
$5 \mathrm{mCi}$ injected Tc99m-Pertechnetate shows normal thyroid uptake of tracer and physiological uptake in the salivary glands (short black arrow). There is a distinct focus of abnormal tracer accumulation in the left parotid/submandibular region. Patient was given lemon juice with evident normal washout from the salivary glands and relative retention by this abnormal focus (long black arrow)

Correlation with laboratory workup, including measurement of serum parathyroid hormone and calcium level, is required. In addition, the evaluating radiologist should search for radiological manifestations of hyperparathyroidism, such as osteopenia, bone resorption, and brown tumours (Fig. 22) $[55,56]$.

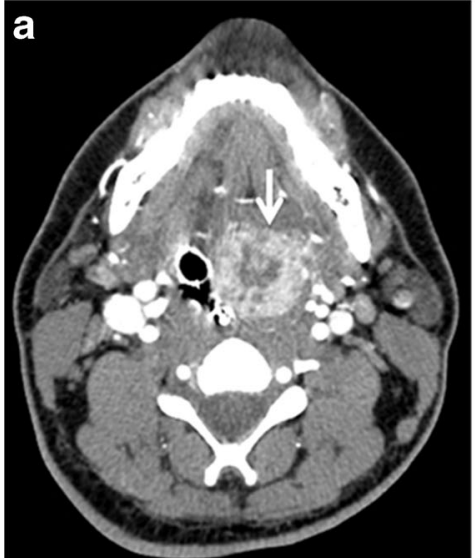

Fig. 18 Lingular thyroid in a 33-year-old male who presented with oropharyngeal bleeding. a Axial enhanced neck CT scan at the level of mandible demonstrates a $3 \times 3 \times 3.4 \mathrm{~cm}$ round, partly well-delineated, heterogeneously enhancing lesion (white arrow). It is predominantly on the left side of the oropharynx and to some extent at the mid part of the base of the tongue. Thyroid gland was normal (not shown). b Image of the

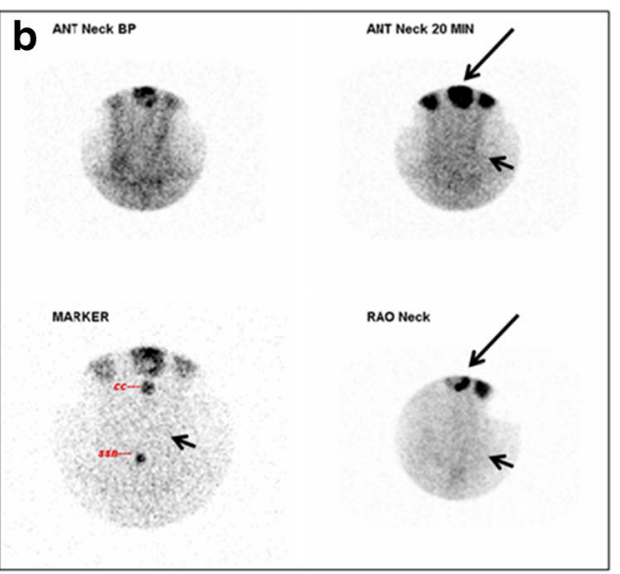

anterior face and neck taken 20 minutes after Tc99m-Pertechnetate injection shows absent thyroid radiotracer uptake in normal thyroid anatomical location (black short arrows). There is an area of increased uptake (long black arrows) corresponding to posterior tongue mass identified on CT scan 

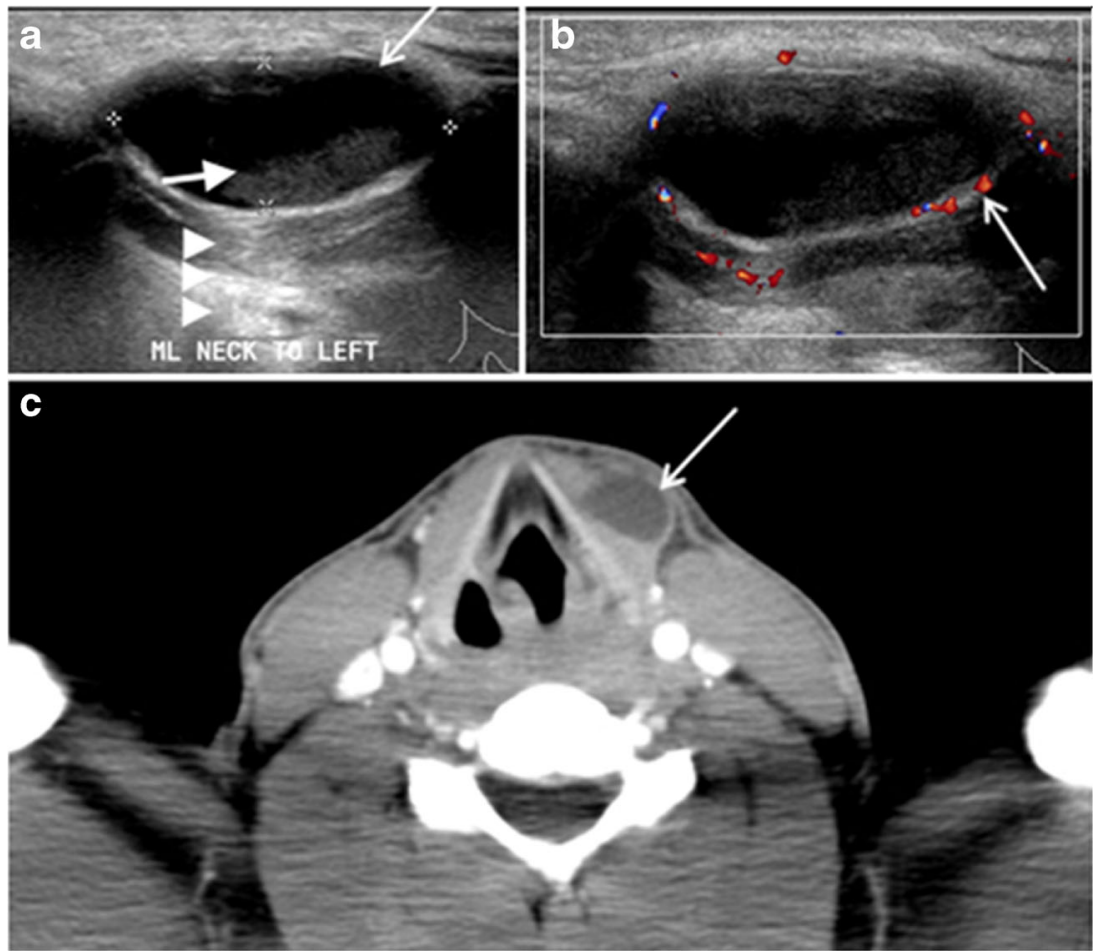

Fig. 19 Long-standing infected thyroglossal duct cyst in a 29-year-old male patient. a Transverse greyscale ultrasound at midline, just above the level of the thyroid gland, shows an oval cystic lesion with internal echoes (white arrows) and posterior enhancement (arrowheads). b Transverse colour Doppler ultrasound shows surrounding peripheral flow (white

In the case of inconclusive Tc99m Sestamibi and neck US imaging, FNA biopsy with FNA-iPTH (intact parathyroid hormone) measurement can provide simultaneous biochemical and cytological evidence. Elevated FNA-iPTH measurement, as compared to serum iPTH, is considered positive and diagnostic of parathyroid adenoma $[57,58]$.
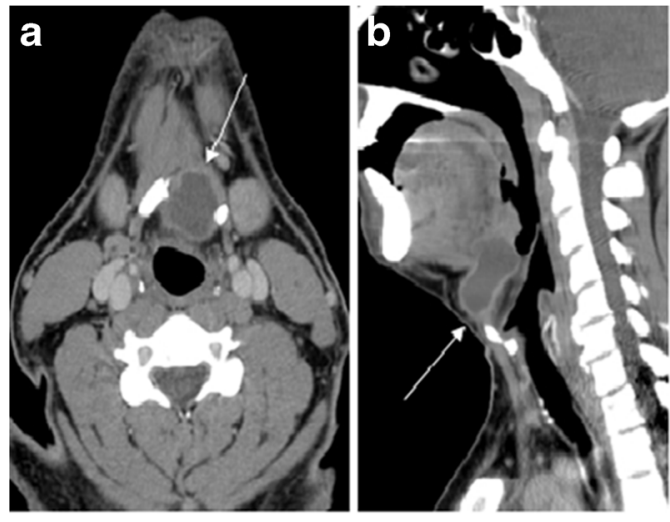

Fig. 20 Recurrent/residual thyroglossal duct cyst in a 39-year-old male patient. The first resection of thyroglossal duct cyst showed histopathology evidence of Hurthle cell type thyroid cancer. However, the second resection showed signs of chronic inflammation, with no malignant cells. a, b Enhanced axial and sagittal neck CT scans demonstrate a unilocular cystic lesion arising from the tongue base and extending through the partially resected hyoid bone. This cystic lesion has arrow). c Axial enhanced neck CT scan at the level of the thyroid cartilage demonstrates a slightly off-midline, well-defined, homogeneous cystic lesion embedded in the left strap muscle with peripheral enhancement (white arrow). It shows no calcification or internal enhancement. Thyroid gland was normal (not shown)

\section{Conclusion}

Thyroid disorders are common and tend to have non-specific appearances on CT scans. Commonly encountered findings when evaluating a CT scan of the neck include thyroid nodules, glandular enlargement, and calcifications.

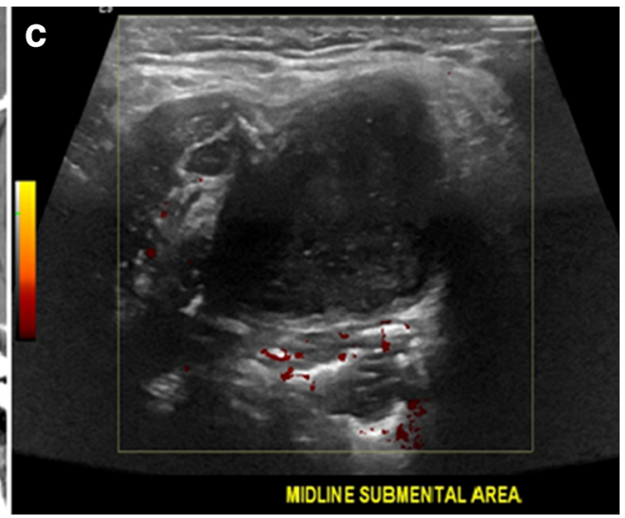

peripheral enhancing wall, which becomes more thick over its inferior aspect associated with surrounding fat stranding at the surgical site (white arrow). There are no internal septations, nodules or masses, or calcifications. c Transverse view of power Doppler ultrasound at the submental area demonstrates cystic lesion and internal debris with no detected internal vascularity 

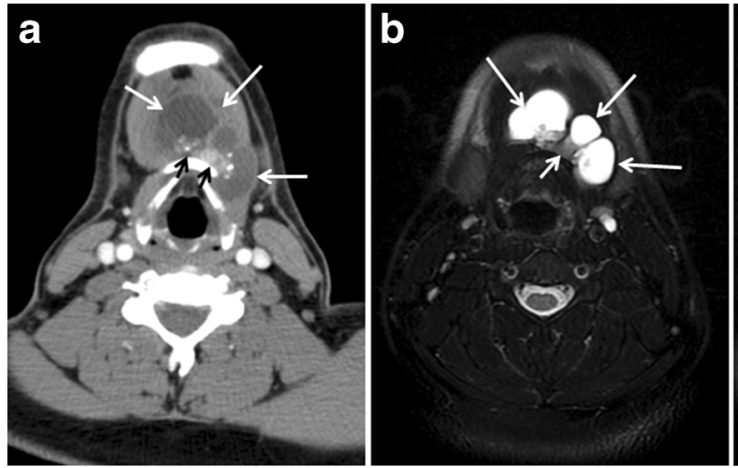

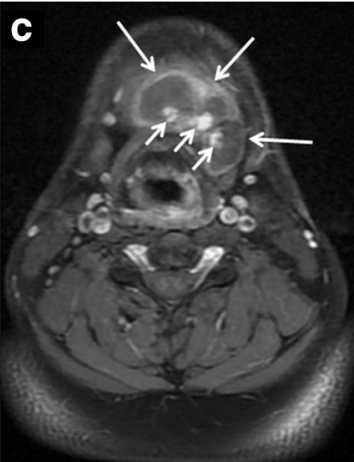

Fig. 21 Papillary thyroid carcinoma arising from a thyroglossal duct cyst in a 28-year-old male. a Axial enhanced CT scan shows a large complex cystic lesion (white arrows) adherent to the anterior aspect of the hyoid bone. It has an enhancing mural solid nodules and calcifications (black arrows). There is no cervical lymphadenopathy. b Axial short tau inversion recovery (STIR) MRI image near the same level shows complex lesion of high signal intensity (long white arrows) with solid mural nodules (short white arrow). c Axial fat saturated T1 MRI image post contrast administration shows the complex cystic lesion with thick enhancing wall (long white arrows) and enhancing mural nodules (short white arrows)
Management of ITNs depends on several factors including nodule size, patient's age, overall health status, and the presence or absence of suspicious features such as lymphadenopathy and/or invasion of adjacent structures.
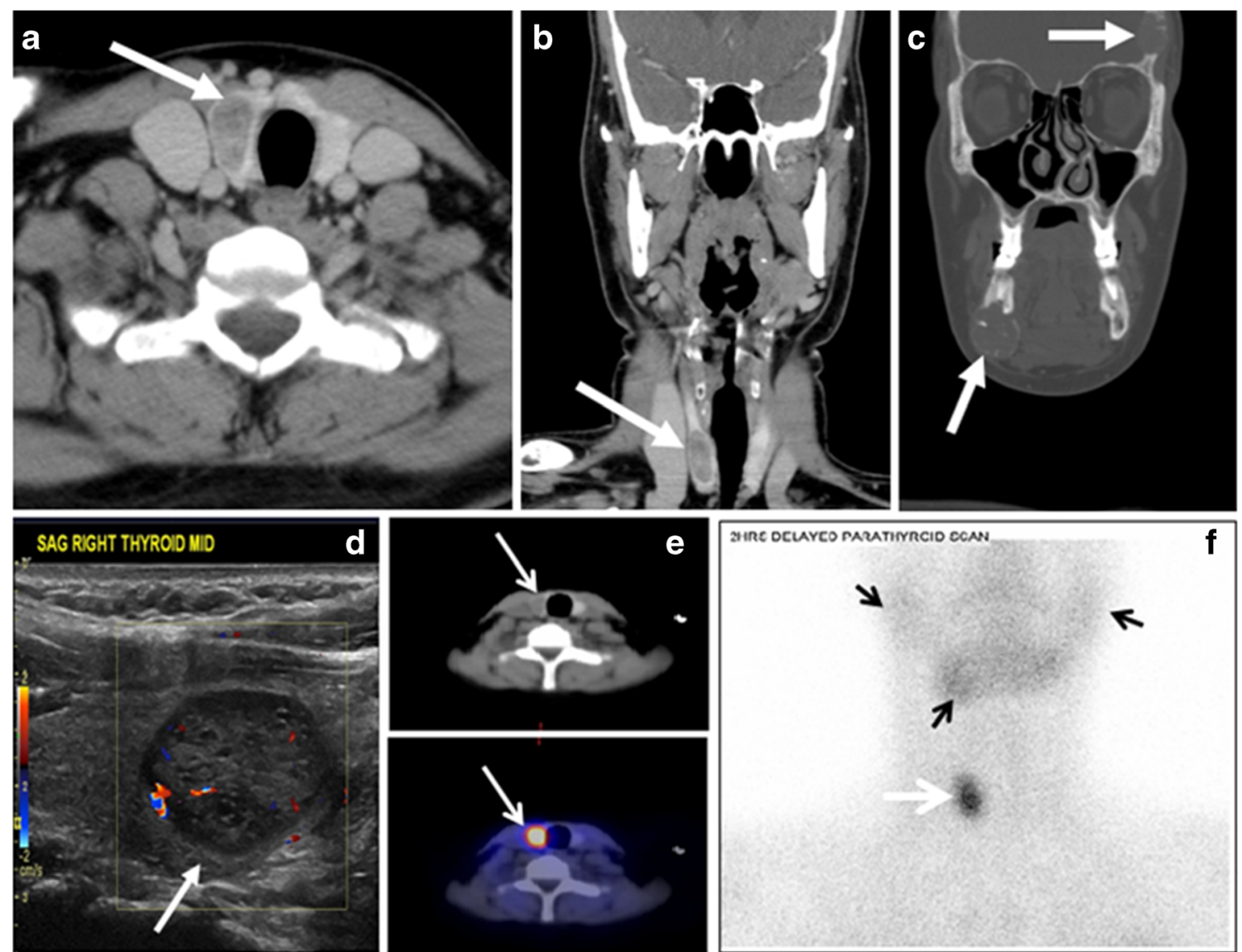

Fig. 22 A 26-year-old male patient with elevated serum parathyroid hormones and calcium secondary to intra-thyroid parathyroid adenoma. a, b Enhanced axial and coronal CT scan of the neck demonstrate a welldefined hypodense right thyroid nodule (white arrows). c Bone window coronal CT scan shows lytic expansile lesions at the right mandible and left frontal bone (white arrows). d Transverse colour Doppler ultrasound of the neck demonstrates a well-defined, heterogonous, predominantly hypoechoic right thyroid nodule measuring $2.7 \mathrm{~cm}$, with mild increased vascularity and no internal micro-calcifications (white arrow). e, $\mathbf{f}$ Delayed anterior planar and fused SPECT/CT parathyroid Sestamibi scan at 2 hours demonstrate persistent focal activity in the right thyroid lobe (white arrows). Note the scattered mandibular/maxillary uptakes in planar image representing the known brown tumours 
A CT scan provides additional important information regarding the local extension of cancer or presence of mass effect, and is useful in evaluating recurrent disease. Furthermore, CT examination plays a crucial role in preoperative evaluation and preoperative surgical planning for patients with symptomatic goiter.

Open Access This article is distributed under the terms of the Creative Commons Attribution 4.0 International License (http:// creativecommons.org/licenses/by/4.0/), which permits unrestricted use, distribution, and reproduction in any medium, provided you give appropriate credit to the original author(s) and the source, provide a link to the Creative Commons license, and indicate if changes were made.

\section{References}

1. Weber AL, Randolph G, Aksoy FG (2000) The thyroid and parathyroid glands. CT and MR imaging and correlation with pathology and clinical findings. Radiol Clin North Am 38(5): 1105-1129

2. Hoang JK, Sosa JA, Nguyen XV, Galvin PL, Oldan JD (2015) Imaging thyroid disease: updates, imaging approach, and management pearls. Radiol Clin North Am 53(1):145-161

3. Iida Y, Konishi J, Harioka T, Misaki T, Endo K, Torizuka K (1983) Thyroid CT number and its relationship to iodine concentration. Radiology 147(3):793-795

4. Hoang JK, Lee WK, Lee M, Johnson D, Farrell S (2007) US Features of thyroid malignancy: pearls and pitfalls. Radiographics 27(3):847-860

5. Wu CW, Dionigi G, Lee KW et al (2012) Calcifications in thyroid nodules identified on preoperative computed tomography: patterns and clinical significance. Surgery 151(3):464-470

6. Shetty SK, Maher MM, Hahn PF, Halpern EF, Aquino SL (2006) Significance of incidental thyroid lesions detected on CT: correlation among CT, sonography, and pathology. AJR Am J Roentgenol 187(5):1349-1356

7. Hoang JK (2014) Reply to "Three-tiered system for incidental thyroid nodules: do not forget the calcifications". AJR Am J Roentgenol 203(4):W453

8. Youserm DM, Huang T, Loevner LA, Langlotz CP (1997) Clinical and economic impact of incidental thyroid lesions found with CT and MR. AJNR Am J Neuroradiol 18(8):1423-1428

9. Nguyen XV, Choudhury KR, Eastwood JD et al (2013) Incidental thyroid nodules on CT: evaluation of 2 risk-categorization methods for work-up of nodules. AJNR Am J Neuroradiol 34(9):1812-1817

10. Yoon DY, Chang SK, Choi CS et al (2008) The prevalence and significance of incidental thyroid nodules identified on computed tomography. J Comput Assist Tomogr 32(5):810-815

11. Hoang JK, Langer JE, Middleton WD et al (2015) Managing incidental thyroid nodules detected on imaging: white paper of the ACR Incidental Thyroid Findings Committee. J Am Coll Radiol 12(2):143-150

12. Kwak JY, Han KH, Yoon JH et al (2011) Thyroid imaging reporting and data system for US features of nodules: a step in establishing better stratification of cancer risk. Radiology 260(3):892-899

13. Frates MC, Benson CB, Doubilet PM et al (2006) Prevalence and distribution of carcinoma in patients with solitary and multiple thyroid nodules on sonography. J Clin Endocrinol Metab 91(9):34113417

14. Lingam RK, Qarib MH, Tolley NS (2013) Evaluating thyroid nodules: predicting and selecting malignant nodules for fine-needle aspiration (FNA) cytology. Insights Imaging 4(5):617-624
15. McQueen AS, Bhatia KS (2015) Thyroid nodule ultrasound: technical advances and future horizons. Insights Imaging 6(2):173-188

16. Haugen BR, Alexander EK, Bible KC et al (2016) 2015 American Thyroid Association management guidelines for adult patients with thyroid nodules and differentiated thyroid cancer: the American Thyroid Association guidelines task force on thyroid nodules and differentiated thyroid cancer. Thyroid 26(1):1-133

17. Hoang JK, Choudhury KR, Eastwood JD et al (2014) An exponential growth in incidence of thyroid cancer: trends and impact of CT imaging. AJNR Am J Neuroradiol 35(4):778-783

18. Hobbs HA, Bahl M, Nelson RC et al (2014) Journal club: incidental thyroid nodules detected at imaging: can diagnostic workup be reduced by use of the Society of Radiologists in ultrasound recommendations and the three-tiered system? AJR Am J Roentgenol 202(1):18-24

19. Ito Y, Miyauchi A, Kihara M, Higashiyama T, Kobayashi K, Miya A (2014) Patient age is significantly related to the progression of papillary microcarcinoma of the thyroid under observation. Thyroid 24(1):27-34

20. Yang J, Schnadig V, Logrono R, Wasserman PG (2007) Fine-needle aspiration of thyroid nodules: a study of 4703 patients with histologic and clinical correlations. Cancer 111(5):306-315

21. Nachiappan AC, Metwalli ZA, Hailey BS, Patel RA, Ostrowski ML, Wynne DM (2014) The thyroid: review of imaging features and biopsy techniques with radiologic-pathologic correlation. Radiographics 34(2):276-293

22. van der Molen AJ, Thomsen HS, Morcos SK (2004) Effect of iodinated contrast media on thyroid function in adults. Eur Radiol 14(5):902-907

23. Luster M, Clarke SE, Dietlein M et al (2008) Guidelines for radioiodine therapy of differentiated thyroid cancer. Eur J Nucl Med Mol Imaging 35(10):1941-1959

24. Yousem DM, Hatabu H, Hurst RW et al (1995) Carotid artery invasion by head and neck masses: prediction with MR imaging. Radiology 195(3):715-720

25. Edge SB (2010) Compton CC The American Joint Committee on Cancer: the 7th edition of the AJCC cancer staging manual and the future of TNM. Ann Surg Oncol 17(6):1471-1474

26. Kebebew E, Clark OH (2000) Differentiated thyroid cancer: "complete" rational approach. World J Surg 24(8):942-951

27. Benbassat CA, Mechlis-Frish S, Hirsch D (2006) Clinicopathological characteristics and long-term outcome in patients with distant metastases from differentiated thyroid cancer. World J Surg 30(6):1088-1095

28. Haq M, Harmer C (2005) Differentiated thyroid carcinoma with distant metastases at presentation: prognostic factors and outcome. Clin Endocrinol 63(1):87-93

29. Kebebew E, Greenspan FS, Clark OH, Woeber KA, McMillan A (2005) Anaplastic thyroid carcinoma. Treatment outcome and prognostic factors. Cancer 103(7):1330-1335

30. Baudin E, Schlumberger M (2007) New therapeutic approaches for metastatic thyroid carcinoma. Lancet Oncol 8(2):148-156

31. Ito Y, Kudo T, Kobayashi K, Miya A, Ichihara K, Miyauchi A (2012) Prognostic factors for recurrence of papillary thyroid carcinoma in the lymph nodes, lung, and bone: analysis of 5,768 patients with average 10-year follow-up. World J Surg 36(6):1274-1278

32. Hay ID, Thompson GB, Grant CS et al (2002) Papillary thyroid carcinoma managed at the Mayo Clinic during six decades (19401999): temporal trends in initial therapy and long-term outcome in 2444 consecutively treated patients. World J Surg 26(8):879-885

33. Pacini F, Schlumberger M, Dralle H, Elisei R, Smit JW, Wiersinga W (2006) European consensus for the management of patients with differentiated thyroid carcinoma of the follicular epithelium. Eur J Endocrinol 154(6):787-803 
34. Ahn JE, Lee JH, Yi JS et al (2008) Diagnostic accuracy of CT and ultrasonography for evaluating metastatic cervical lymph nodes in patients with thyroid cancer. World J Surg 32(7):1552-1558

35. Choi JS, Kim J, Kwak JY, Kim MJ, Chang HS, Kim EK (2009) Preoperative staging of papillary thyroid carcinoma: comparison of ultrasound imaging and CT. AJR Am J Roentgenol 193(3):871878

36. Lesnik D, Cunnane ME, Zurakowski D et al (2014) Papillary thyroid carcinoma nodal surgery directed by a preoperative radiographic map utilizing CT scan and ultrasound in all primary and reoperative patients. Head Neck 36(2):191-202

37. Johnson NA, Tublin ME (2008) Postoperative surveillance of differentiated thyroid carcinoma: rationale, techniques, and controversies. Radiology 249(2):429-444

38. Lee DH, Kang WJ, Seo HS et al (2009) Detection of metastatic cervical lymph nodes in recurrent papillary thyroid carcinoma: computed tomography versus positron emission tomographycomputed tomography. J Comput Assist Tomogr 33(5):805-810

39. Leboulleux S, El Bez I, Borget I et al (2012) Postradioiodine treatment whole-body scan in the era of 18 -fluorodeoxyglucose positron emission tomography for differentiated thyroid carcinoma with elevated serum thyroglobulin levels. Thyroid 22(8):832-838

40. Rosario PW, Mourao GF, dos Santos JB, Calsolari MR (2014) Is empirical radioactive iodine therapy still a valid approach to patients with thyroid cancer and elevated thyroglobulin? Thyroid 24(3):533-536

41. Wilhelm SM, Robinson AV, Krishnamurthi SS, Reynolds HL (2007) Evaluation and management of incidental thyroid nodules in patients with another primary malignancy. Surgery 142(4):581586

42. Rios A, Rodriguez JM, Galindo PJ, Montoya MJ, Canteras M, Parrilla P (2005) Surgical treatment of multinodular goiter in young patients. Endocrine 27(3):245-252

43. Pollard DB, Weber CW, Hudgins PA (2005) Preoperative imaging of thyroid goiter: how imaging technique can influence anatomic appearance and create a potential for inaccurate interpretation. AJNR Am J Neuroradiol 26(5):1215-1217

44. Jhaveri K, Shroff MM, Fatterpekar GM, Som PM (2003) CT and MR imaging findings associated with subacute thyroiditis. AJNR Am J Neuroradiol 24(1):143-146

45. Zander DA, Smoker WR (2014) Imaging of ectopic thyroid tissue and thyroglossal duct cysts. Radiographics 34(1):37-50
46. Noussios G, Anagnostis P, Goulis DG, Lappas D, Natsis K (2011) Ectopic thyroid tissue: anatomical, clinical, and surgical implications of a rare entity. Eur J Endocrinol 165(3):375-382

47. Altay C, Erdogan N, Karasu S et al (2012) CT and MRI findings of developmental abnormalities and ectopia varieties of the thyroid gland. Diagn Interv Radiol 18(4):335-343

48. Caccetta TP, Kumar A (2005) Ishak H Lateral aberrant thyroid tissue presenting as a lateral neck mass. ANZ J Surg 75(12): 1123-1124

49. Prado H, Prado A, Castillo B (2012) Lateral ectopic thyroid: a case diagnosed preoperatively. Ear Nose Throat J 91(4):E14-E18

50. Batsakis JG, El-Naggar AK, Luna MA (1996) Thyroid gland ectopias. Ann Otol Rhinol Laryngol 105(12):996-1000

51. Pelizzo MR, Torresan F, Grassetto G, Briani G, Marzola MC, Rubello D (2011) Imaging identifies submandibular ectopic thyroid tissue. Clin Nucl Med 36(8):728-730

52. Lingam RKNF, Nigar E, Kalan A (2011) Chronic lymphocytic thyroiditis presenting as a jugulodigastric neck mass. Otorhinolaryngologist 3(3):118-120

53. Mysorekar VV, Dandekar CP, Sreevathsa MR (2004) Ectopic thyroid tissue in the parotid salivary gland. Singap Med J 45(9):437438

54. Yang YJ, Haghir S, Wanamaker JR, Powers CN (2000) Diagnosis of papillary carcinoma in a thyroglossal duct cyst by fine-needle aspiration biopsy. Arch Pathol Lab Med 124(1):139-142

55. Goodman A, Politz D, Lopez J, Norman J (2011) Intrathyroid parathyroid adenoma: incidence and location-the case against thyroid lobectomy. Otolaryngol Head Neck Surg 144(6):867-871

56. Silaghi H, Valea A, Ghervan C, Silaghi AC (2011) Ectopic intrathyroid parathyroid adenoma: diagnostic and therapeutic challenges due to multiple osteolytic lesions. Case report. Med Ultrason 13(3):241-244

57. Abraham D, Sharma PK, Bentz J, Gault PM, Neumayer L, McClain DA (2007) Utility of ultrasound-guided fine-needle aspiration of parathyroid adenomas for localization before minimally invasive parathyroidectomy. Endocr Pract 13(4):333-337

58. Kwak JY, Kim EK, Moon HJ et al (2009) Parathyroid incidentalomas detected on routine ultrasound-directed fine-needle aspiration biopsy in patients referred for thyroid nodules and the role of parathyroid hormone analysis in the samples. Thyroid 19(7):743-748 